\title{
Molecular and microstructural biomarkers of neuroplasticity in neurodegenerative disorders through preclinical and diffusion magnetic resonance imaging studies
}

Rodolfo Gabriel Gatto ${ }^{1, *}$

${ }^{1}$ Department of Bioengineering, The University of Illinois at Chicago, Illinois, 60607, USA

*Correspondence: rgatto@uic.edu (Rodolfo Gabriel Gatto)

DOI: $10.31083 /$ i.jin.2020.03.165

This is an open access article under the CC BY 4.0 license (https://creativecommons.org/licenses/by/4.0/).

Advances in the understanding of genetic and molecular mechanisms and imaging technologies have opened a new window of research possibilities to address dynamic processes associated with neuroplasticity in physiologically intact models of neurodegenerative diseases. This review aims to: (i) establish the most relevant molecular mechanisms, as well as cellular and structural biomarkers in the study of neuroplasticity; (ii) introduce different neurodegenerative diseases in animal models that contribute to our knowledge of neuroplasticity; and (iii) illustrate the capabilities and limitations of current diffusion magnetic resonance imaging techniques to study cortical plasticity, as well as the use of alternative diffusion models.

\section{Keywords}

Neuroplasticity; neurodegenerative diseases; preclinical animal models; bioimaging markers; axonal degeneration; diffusion magnetic resonance imaging; diffusion tensor imaging; anomalous magnetic resonance imaging diffusion

\section{Introduction}

Upon the early $20^{\text {th }}$ century, the rendition of the original Spanish works of 1899-1904 and French works of 1909-1911, Cajal (2002) already described in his publication structural modifications that imply the formation of new neural pathways through ramification and progressive growth of the dendritic terminals (see Stahnisch and Nitsch 2002 and Lobato 2008). However, for many decades, the human central nervous system (CNS) was considered anatomically fixed and incapable of undergoing selfmodification. This view assumed that no new neurons were created. In this paradigm, the CNS remained static after completion of its embryonic patterning, and eventual lesions or degenerative processes were considered as irreversible damage. However, in recent decades, this dogma has been challenged; from a rigid and invariant number of neuronal units and circuits, towards a more dynamic connectivity model constantly adapting to relentless environmental changes (Murphy et al., 2014). The lifelong capacity of the brain to change and rewire itself in response to stimulation and "learning by experience" is called neuroplasticity (NP) and was introduced by Polish neurophysiologist Jerzy Konorski in
1948 (Bijoch et al., 2020). This is based on the formation of new functional and structural communication processes and could be the base of therapeutic strategies to reorganize recovery and eventually repair the loss of functions from neuronal damaging events. Logically, the NP process is intricately related to the brain's ability to create new neurons and connections throughout a lifetime, so-called neurogenesis (NG). Adult NG is known to occur in the hippocampus, amygdala, and lateral ventricles of the mammalian brain.

In general terms, NP can be evaluated as part of the initial organism stage (developmental neuroplasticity) or as a process occurring during the mature form (adult neuroplasticity), the last concept initially described by Gould (1999). Molecular and cellular mechanisms of NP phenomena can be classified into other sorts: functional NP, which includes changes in the efficacy of synaptic transmissions such as long-term potentiation and the activation of silent synapses; and structural NP, associated to changes in cell structure, as well as mechanisms of axonal regeneration, collateralization and reactive synaptogenesis (BergadoRosado and Almaguer Melián, 2000). Both forms of plasticity are interconnected. The functional activity drives the formation of new morphological features, such as creating more nodes and hubs for information storage and exchange, enhancing the functional capacity of the network.

To facilitate our understanding of the complex NG process, molecular and cellular methodologies have been developed to recapitulate some NP components in living organisms, decreasing the structural complexity of the entire biological systems. This shortened approach has been fundamental to understand some of the underlying mechanisms in neuronal cell development, biology, function, and cell-to-cell interaction (Schlachetzki et al., 2013). For example, in vitro models to study embryonic neurogenesis and the formation of different neuronal phenotypes are mainly based on the usage of pluripotent stem cells, such as embryonic stem cells (ESCs). Up to date, three culture systems can generate neural cells from the pluripotent stem cells, which include: embryoid body cells, cell co-cultures (such as bone marrow stromal cells or their conditioned medium that potentiates the neuralization processes), and culture monolayer systems. Thus, cell culture models of adult neurogenesis are mainly based on the use of fetal, post- 
natal, and adult neural stem cells (NSCs). However, the study of $\mathrm{NP}$ on in vitro models is limited to recapitulate the real complex events occurring in intact physiological organisms significantly to restore damaged neuronal connectivity in the mammalian brain.

Carefully selected to answer specific scientific questions, animal models have been among the most important tools in the study of NP processes. This is particularly true in mammals due to their similitude with the human brain (La Rosa and Bonfanti, 2018). In the adult brain, neurogenesis occurs across very well-defined areas of the CNS in the presence of undifferentiated stem cells in very sophisticated organized structures systems referred to NSC niches (Decimo et al., 2012). Structural changes are observable in superficial and deep CNS structures, continually changing during dynamic processes such as cognitive functions. This establishes the basis for higher functions, which is accomplished by inputs from a rich sensory environment generated by biological and nonbiological sources (Krubitzer and Dooley, 2013) and genes that shape the developing neocortex. Thus, the modification of intellectual capacities by regulating existing synapses can eventually contribute to the functional compensation of silent or low activated synapses, which are the foundation of the so-called behavioral plasticity, discussed further in this review. At the subcellular level, similar synaptic and connectivity changes in neuronal dendrites and spines can be considered fundamental physiological processes in learning and memory. Some of these mechanisms are embedded in the functional maturation process of the cerebral cortex formation. In contrast, other alterations in adult neurogenesis appear to have a commonality with the events leading to different neurodegenerative diseases (NDDs) (Winner et al., 2015).

Despite the intensive research in neurobiology and medical sciences, NDDs including dementias, and mental illnesses, remain as incurable illnesses with no effective therapies able to modify their course. The main constraint of NP as an alternative therapy in NDDs is strictly linked to a well-known feature of the mammalian nervous tissue; its scarce capacity of renewing the cellular components through adulthood and undergo efficacious reparative processes after cellular damage (La Rosa and Bonfanti, 2018). Current attempts of therapeutic interventions by NG modulation in the clinical field (Cramer et al., 2011) face significant challenges due to many factors; how to generate neurons to replenish the neuronal loss caused by injuries or neurodegeneration (neuronal plasticity), how to promote and recover the loss in cellular connectivity ( $a x$ onal plasticity), or how to limit the secondary tissue damage or scars caused by the long-term accumulation of glial cells at the injury site (glial plasticity), among many other pathological events.

Magnetic resonance imaging (MRI), due to its superior spatial resolution, is a powerful neurobiological imaging tool able to extract functional and structural information from the entire CNS (Hamaide et al., 2016). Its non-invasive character, along with its dependence on magnetism instead of ionizing radiation or radioactive substances, allows dynamic longitudinal in vivo studies that can follow upon maturation, aging, genetic manipulation, motor, and behavioral training. Combining various imaging techniques, MRI makes it possible to extract various structural information at the macro and microanatomical level. As a structural imaging modality, diffusion magnetic resonance imaging (dMRI) is based on the preferential molecular Brownian motion (diffusion) of wa- ter molecules limited by the local microarchitecture of the brain. Thus, dMRI techniques measure the diffusion profile of water, making this imaging modality ideal to study white matter (WM) or grey matter (GM) macroscopic or microscopic features, such as dendrite branching, cellular density, and cellular shape (Ashburner and Friston, 2000; Tardif et al., 2016; Wang et al., 2015). Diffusion parameters indirectly linked to glial activity (myelinization) and probabilistic tractography maps are among many other potentially useful bioimaging markers related to the structural biological remodeling events occurring during NP mechanisms.

Overall, each component of this adaptative system plays an interconnected part in various responses to different environmental factors. In the following sections, an examination of different molecular constructs and mechanisms will be provided. Such biochemical tags are the foundation of the micro and macrostructural biomarkers related to cortical plasticity. In the last sections, we will provide a further assessment of how such histological descriptors can vary in the context of different preclinical animal models of NDDs and the application of dMRI techniques to study the interconnected flexible structural and functional phenomena in intact physiological organisms (Fig. 1). In the end, the combination of different molecular, neuropathological, and imaging technologies will provide a further understanding of how the NP mechanisms operate, with a better application of this knowledge in the discovery of new neuroregenerative therapies in patients with NDDs.

\section{General molecular mechanisms of neuroplasticity}

The mechanisms of neuroplasticity are diverse and constitute an extensive combination of different biological features, from external morphological modifications, such as regeneration of an axon or synaptic processes, to minimal molecular, synaptic changes leading to a different cellular response to neurotransmitters (Gulyaeva, 2017). Different epigenetic factors can modulate the temporal dynamics of the NP processes. At the same time, other endogenous mediators can alter the course of NP by direct or indirect interaction with key regulatory molecular pathways $(\mathrm{Fa}-$ giolini et al., 2009). In this section, an overview of the most common molecular mediators and molecular biomarkers in the field of NP is described, which represents the fundamental blocks of microstructural and biomarker changes described in other sections.

\subsection{Glutamate receptors}

There are two types of glutamate receptors: 1) Ionotropic receptors, ionic channels responsible for the postsynaptic membrane depolarization. Among those, the most commonly described are the $\alpha$-amino-3-hydroxy-5-methyl-4-isoxazole propionic acid (AMPA) and the N-methyl-D-aspartate (NMDA) receptors (named after its agonist); 2) The so-called metabotropic receptors, a family of $\mathrm{G}$ protein-coupled receptors. Among them, the AMPA/kainate receptors are responsible for the synaptic transmission mediating the sodium currents, which depolarize the postsynaptic membrane. However, when the level of depolarization reaches a threshold level in the presence of glutamate, magnesium is released from the NMDA receptor following its activation. Then, the NMDA receptor opens the calcium $\left(\mathrm{Ca}^{2+}\right)$ associated channel, a decisive event in the production of a long-term potentiation (LTP) (a long-lasting and activity-dependent event), which 


\section{Biomarkers of Neuroplasticity}

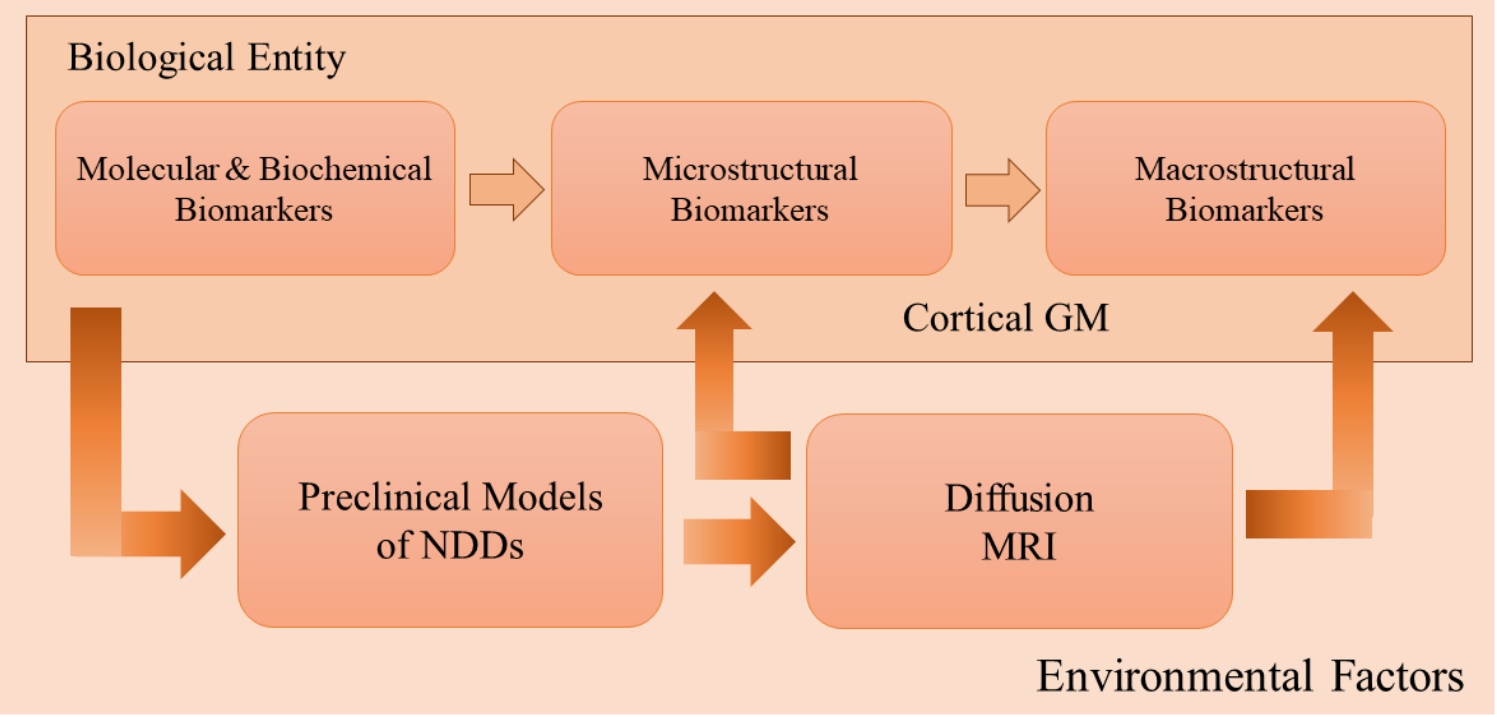

Fig. 1. Relationship between different biological and bioimaging markers of neuroplasticity. Molecular mechanisms represented by diverse biochemical biomarkers (described in section 2) constitute the fundamental biological building blocks in developing an adaptative response to different environmental factors. Microstructural (section 3) and macrostructural biomarkers, specifically in grey matter (GM) structures (described in section 4), provide an increased level of complexity that can be mainly studied in different preclinical models of NDDs (section 5). Diffusion MRI is a useful tool to examine macro and microstructural physiological events associated with structural bioimaging markers in NDDs preclinical models (section 6). Such studies can be applied to neuropathological and imaging clinical settings. Abbreviations: GM, grey matter; NDDS, neurodegenerative diseases; MRI, magnetic resonance imaging.

increases the strength of the synaptic transmission. As the NMDA receptor allows the intracellular increase of $\mathrm{Ca}^{2+}$, the surge of LTP increases the density of the AMPA and NMDA receptors in the membrane (Bashir et al., 1991; Mattson, 2008; Michaelis, 1998).

\subsection{Magnesium}

One significant action of magnesium $\left(\mathrm{Mg}^{2+}\right)$ is modulating the voltage-dependent block of N-Methyl- d-aspartate (NMDA) receptors (NMDAR), controlling their opening during coincidence detection that is critical for synaptic plasticity. $\mathrm{Mg}^{2+}$ affects many biochemical mechanisms that are vital for neuronal functions. Moreover, an increase in brain magnesium enhances both short-term synaptic facilitation and long-term potentiation improving learning and memory functions. Interestingly, recent studies have demonstrated that magnesium treatment protects cognitive function and synaptic plasticity by inhibiting GSK-3 $\beta$ in sporadic Alzheimer's disease (AD) model rats (Xu et al., 2014). As such, $\mathrm{Mg}^{2+}$ ions are a critical factor in controlling synapse density and plasticity, showing a reduction in $\mathrm{A} \beta$-plaques and cognitive deficits in APPswe/PS1dE9 mice, a transgenic mouse model of $\mathrm{AD}$ (Li et al., 2014). However, the effects of dietary $\mathrm{Mg}^{2+}$ deficiency on learning and memory are not followed by changes in the spine density and morphology of hippocampal neurons of rodent models (Serita et al., 2019), and further examination of this topic is undoubtedly needed.

\subsection{Calcium channels}

The influx of calcium $\left(\mathrm{Ca}^{2+}\right)$ in the postsynaptic terminal is a necessary condition to initiate the synaptic potentiation pro- cesses. The increase of $\mathrm{Ca}^{2+}$ is usually associated with an increase in the permeability of the NMDA receptors, also obtained by the activation of voltage-dependent $\mathrm{Ca}^{2+}$ channels (VDCC). Thus, $\mathrm{Ca}^{2+}$ acts as a second messenger in membrane voltage mechanisms (such as LTP) and long-term depression (LTD). Recently, it has been implied that $\mathrm{Ca}^{+2}$ T-type channels participate in numerous homo- and hetero-synaptic plasticity mechanisms that involve different molecular partners and both pre- and postsynaptic modifications. In contrast, other studies have focused on the adaptive activities within cerebellar circuits (LTD and LTP at parallel fiber-Purkinje cell synapses), potentially crucial to cerebellar motor learning. In the generation of such electrical events, a particular role has been described for P/Q-type calcium channels and downstream small-conductance calcium-sensitive potassium channels (SK2) effectors regulation of intrinsic excitability and synaptic plasticity (Liu and Friel, 2008).

\subsection{Protein-kinases}

Phosphorylation of proteins is an essential mechanism of biomolecular regulation by enzymes known as protein kinases directly affecting the synaptic NP. Increment of $\mathrm{Ca}^{2+}$ activates protein kinases, maintaining the transient status and the synaptic transcription/translation processes. Also, some of these protein kinases can modulate the $\mathrm{Ca}^{2+}$ reservoirs in mitochondria and endoplasmic reticulum, augmenting the liberation of the cytoplasm and increasing its intercellular concentration. Tyrosine-protein kinases are activated (NMDA and VGCC mechanisms) and modulated by $\mathrm{Ca}^{2+}$ ions and other enzymes such as Calmodulin, us- 
ing multiplicative intracellular mechanisms and further potentiation of the postsynaptic channels. Other protein kinases (PKA and $\mathrm{PKC})$ also have an influential role in the synaptic efficiency, phosphorylating, and modulating pre- and postsynaptic ionic channels (Geribaldi-Doldán et al., 2019).

\subsection{Neurotrophic factors}

Nerve growth factors (NGF) are molecules of natural origin that stimulate and promote survival and neural cell development (Binder and Scharfman, 2004). There are a variety of other molecules with neurotrophic capabilities. Generally, neurotrophic factors or neurotrophins (NTF) can be classified in different families based on the degree of molecular similarities and the receptor type used to obtain their trophic effects. Interestingly, many of these molecules have a high degree of phylogenetic conservation, making quite evident their biological importance and critical roles in neuron survival. Many of them are described in presynaptic terminals and allocated to the soma by retrograde transport. Such a continuous supply of these factors is vital to keep the cellular function in neuronal cells. It appears to be involved in programmed cell death (apoptotic mechanisms) during different stages of neurodevelopment.

There are four prominent NTFs characterized in mammals: Nerve growth factor (NGF), brain-derived neurotrophic factor (BDNF), neurotrophin-3 (NT-3), and neurotrophin-4 (NT-4), all derived from a common ancestral gene and similar in sequence and structure (Huang and Reichardt, 2001). NTFs produce their effects by membrane receptors connecting different intracellular molecular pathways such as Mitogen-activated protein kinase (MAPK), protein kinase $\mathrm{C}$ (PKC), and phosphatidylinositol 3-Kinase (PI3$\mathrm{K})$, can modify the genetic expression and protein synthesis (Morfini et al., 2013; Sama et al., 2017). Hence, such molecules can induce and modulate different functional mechanisms of neuroplasticity regulating cellular growth. Other NTFs with neurotrophic properties are epidermal growth factor (EGF), insulinlike growth factor 1 (IGF-1), vascular endothelial growth factor (VEGF), fibroblast growth factor (FGF) and platelet-derived growth factor (PDGF), etc., that can activate similar molecular cascades. Additionally, other studies are focused on Glial CellLine Derived Neurotrophic Factor (GDNF), a member of the NTF family of ligands (GFLs) that also includes neurturin (NRTN), artemin (ARTN), and persephin (PSPN) (Saarma and Sariola, 1999). GFLs play a pivotal role in several biological processes, including cell survival, neurite outgrowth, cell differentiation \& migration. Overall, the role and possible therapeutic effects of these molecules in cortical plasticity are still under investigation. Other factors affecting neuronal growth and regeneration of axonal or dendritic processes; the ciliary neurotrophic factor (CNTF), a protein mainly located in the olfactory epithelium of rodents and synthetized by glial cells, has been linked to the rescue of several types of neurons from axotomy-induced death (Richardson, 1994). However, the roles of CNFT on neuroplasticity mechanisms and its potential clinical applications are still to be determined.

In deep GM brain structures such as the hippocampus, the production of NTF is linked to the voltage-dependent mechanism. Reversely, the administration of NTF, such as BDNF, can produce an LTP response. Contrariwise, LTP induced several NTF mechanisms: phosphorylation of the NMDA receptor, the activation of protein kinase and the synthesis of proteins, and the retrograde signaling due to induced presynaptic changes and mechanisms involving MAPK. Additionally, NTF can support synaptic neuroplasticity processes and strengthen the influence of modulatory glutamatergic pathways to modulate LTP. NTFs have an important role in the cortical NP, leading to an experience-dependent functional maturation of thalamocortical circuits with afferents in the visual and somatosensorial neuronal pathways, a key factor during critical stages of neurodevelopment. Further, experimental evidence suggests the neuroprotective effect of NTFs against neuronal damage by activating enzymatic systems of cellular defense (Garcia et al., 2010).

\subsection{Gangliosides}

The gangliosides are glycosphingolipids that contain sialic acid, and they are found in significant concentration in synaptic membranes (Curtis et al., 2011). In vitro studies have shown these molecules exhibit neuroprotective and neuro proliferative properties and have been considered beneficial in CNS and peripheral nervous system (PNS) repair (Schnaar, 2019). It has been also demonstrated that the administration of gangliosides in animal models could improve memory by LTP mechanisms. However, some of these results are still questioned. Gangliosides have also shown protective effects over dopaminergic, cholinergic, serotoninergic, and noradrenergic neurons (among others) in animal models. Unfortunately, gangliosides can only be administered by an intravenous route because they are destroyed in the digestive tract from where only a tiny fraction can reach the brain. Despite pharmacokinetic and pharmacodynamic limitations, gangliosides are still part of a potentially useful pharmacological group, particularly as a neuromodulator of NG in neurodegenerative pathologies (Magistretti et al., 2019).

\subsection{Steroids}

Steroids can be divided into glucocorticoids, mineralocorticoids, and sex hormones. Their mechanisms of action are based on its interaction with an intranuclear protein receptor accessing the DNA, promoting the transcription and synthesis of proteins. Due to its lipophilic chemical properties, these steroid hormones can penetrate the brain-blood-barrier and cellular membranes, conferring these molecules' genomic effects and membrane receptor extra-genomic effects. Henceforth, nuclear steroids receptors localized in multiple brain areas (limbic system, the hippocampus, and hypothalamus) are instead of and possibly one of the main molecules to modulate NP pathways (Sheppard et al., 2019).

Glucocorticoids (GR) are the principal stress hormones demonstrating different effects in terms of NG mechanisms. Conversely, in models of the lesioned entorhinal cortex, the direct administration of corticosterone reduces reinnervation and synaptogenesis. These anti-NG effects occur independently of the negative regulation by the glucocorticoid receptor during the differentiation processes and vanish with the aging process. Mechanisms through membrane receptors have been discovered for molecules (such as pregnenolone) to produce its effects (Smith et al., 2014). Further, the balance between the actions of mineralocorticoid (MR) and GR receptors in some areas of the brain (limbic system) is critical for neuronal activity, behavioral programming, and adaptation. Overall, MR appears to promote pro-survival effects, 
while exclusive GR activation favors neurodegeneration. Yet, the sustained co-activation of both receptors in chronic stress scenarios generally results in moderate effects, such as dendritic atrophy and impaired synaptic plasticity (Sousa et al., 2008).

Sex hormones, such as estrogens, also have neurogenic effects. However, (in some cells) its effects are only restricted to axonal growth. In the hippocampus, their prominent actions seem to be related to the augmentation of dendritic spines density (based on an enhanced NMDA mechanism), stimulating the formation of new synapses over preexistent terminal buttons. In these brain regions, estrogens stimulate the mechanisms of synaptic NP and neuronal growth by a related AMPc-PKA-CREB molecular pathway. The high sensitivity of the estrogens to the mechanisms of $\mathrm{NP}$ can be validated in experiments involving female rats along the different stages of their hormonal cycle; NP is enhanced when the hormonal concentration is elevated and lowered when hormonal levels decline. In animal models like songbirds, Soma et al. (2004) discovered a dramatic NP large-scale anatomical changes in discrete brain regions called "song control nuclei" (SCN) mediated by an increase in the sex hormone testosterone and mediated by neural conversion to estradiol (E2) by the enzyme aromatase. This study demonstrates that estrogen can affect adult neural plasticity on a gross anatomical scale in the whole animal brain (Soma et al., 2004).

\subsection{Environmental factors}

Environmental factors are critical components to enhance NP in living organisms. Generally, a subtle interplay exists between sensory experience and innate genetic programs, leading to the sculpting of neuronal circuits during the early stages of brain development. This suggests a dynamic mechanism of regulation in gene expression between epigenetic mechanisms and environmental stimuli, leading to molecular, cellular, and complex behavioral phenotypes. A growing body of research has been conducted to link environmental effects such as caloric intake, environmental enrichment, and exercise training to enhance synaptic \& dendritic plasticity and axonal rewiring and sprouting. Remarkably, particular environments like microgravity have also been reported having an impact on human NP (Van Ombergen et al., 2017). Thus, manipulating environmental factors is one of the most common and more straightforward therapeutics approaches in structural and functional neuro-regeneration, enhancing neuro repair to diminish the damage and modulate brain health and function (Mandolesi et al., 2017; Voss et al., 2013).

\section{Microstructural neuroplasticity}

One of the unique properties of the nervous system is the usedependent rearrangement of its neural circuits. CNS structure and function are susceptible to changes induced by prior neuronal activity reflected by brief and lasting cellular microstructural modifications, which leads to improvements in synaptic efficiency as well as neuronal excitability (Zhang and Poo, 2010).

\subsection{Neuronal plasticity (neurogenesis)}

The cortex is the part of the brain involved in several high intellectual functions such as perception, cognition, and volitional motor control. This highly dynamic structure is dramatically altered within the lifetime of biological organisms (Krubitzer and Dooley, 2013). Combining genetic and environmental factors account for the remarkable variations in behavior that different animal species exhibit during their development. Thus, several changes in the cortical organization (absolute and relative size of the cortical sheet, relative size, number, connections addition of modules of cortical fields, among many others) are part of the constant neurogenesis dependent structural modifications to adapt in a shifting environment.

First described in primates (macaque monkey), the outer subventricular zone (OSVZ) (Smart, 2002) was a population of proliferative radial glia-like cells termed outer radial glial cells (oRG). These neurons populate the cerebral cortex, promoting exponential growth during the neuro-proliferative events in large-brained gyrencephalic mammals. A much smaller OSVZ has been described in lissencephalic brained rodents as well, able to generate expansions across specific cortical sheets (Martínez-Cerdeño et al., 2012). Remarkably, mounting evidence suggests that changes in the size of the cortical sheet are due to expansions of preexisting cell populations based on cell-cycle kinetics, rather than the creation of new cell lineages. In deep GM structures such as the hippocampus, the dentate gyrus (DG) is a key epicenter of neurogenesis. This structure is (among other functions) responsible for the formation of new episodic memories and the spontaneous exploration of novel environments. Thus, newly born DG's granule cell neurons must become wired into a neural network by forming synapses via NP to stay alive and participate in the ongoing neural circuit function (Fig. 2a).

\subsection{Synaptic plasticity}

In general terms, increased efficiency in synaptic exchange can be obtained by; a) a surge in the amount of neurotransmitter in the synaptic terminal, b) a rise in the postsynaptic receptor's affinity for the neurotransmitter, and c) increase density of postsynaptic receptors. Although recent evidence points to an increase in the release of glutamate after LTP, this has not been demonstrated. Indirect evidence such as the increase of vesicular fusion related proteins and release of neurotransmitter hours after the LTP induction seems to support these events. Related observations in the hippocampus pointed towards a link between LTP and an increase in the synaptic transmission. The presynaptic component of the LTP requires the activation of postsynaptic neurons. Thus, it has been postulated that the postsynaptic neurons release a messenger activating the presynaptic terminals to produce its synaptic effects. Among the discovered messenger molecules, several mediators (adenosine, BDNF, nitric oxygen (NO), carbon monoxide (CO), platelet activator factor (PAF), arachidonic acid, etc.) have been proposed.

A repetitive number (high frequency) of synaptic events are required to induce a long-lasting LTP to simultaneously activate a significant number of fibers. Although the NMDA receptor has a significant cooperative role in the development and maintenance of the LTP, it can also be modulated by non-glutaminergic synapses. As an example, the administration of muscarinic agonists can induce an enhancement in the late stages of the LTP. Likewise, the administration of dopaminergic antagonists halts the LTP at late stages and, the administration of agonist induces a retarded potentiation response. In this scenario, dopamine seems to act upon the activation of a phosphorylated protein kinase. Other experiments have proven that sodium $\left(\mathrm{Na}^{+}\right)$depletion may af- 

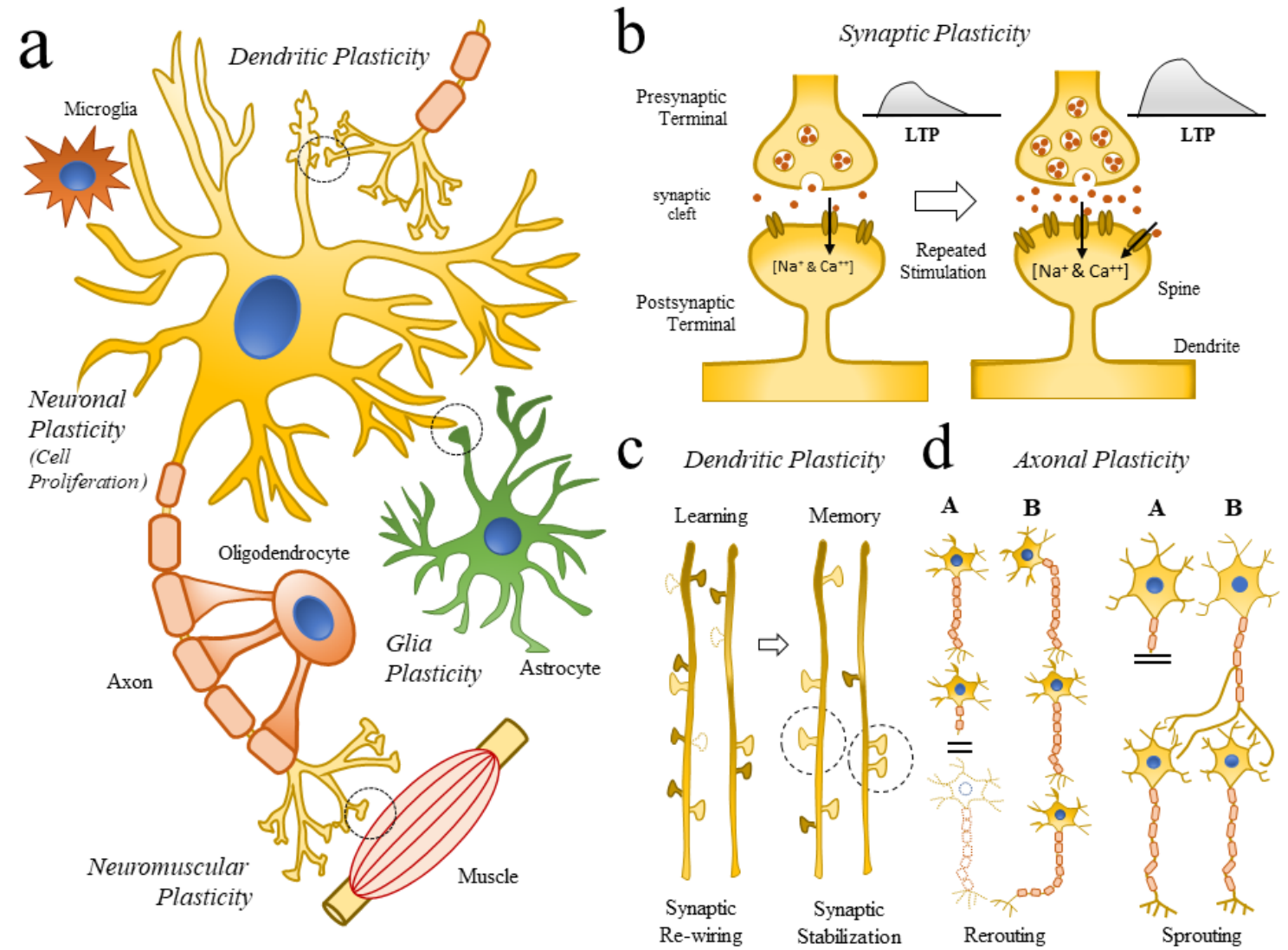

Fig. 2. Changes in cellular structures related to neuroplasticity. (2a) Schematic representation of key cellular elements (neuronal and glial cells) involved in the neuroplasticity (NP) process (Neuronal and Glia plasticity) as well as subcellular compartments (Synaptic, Dendritic, Axonal, Neuromuscular Plasticity). (2b) Schematic diagram representing how repetitive synaptic stimulations repetitive LTPs are linked to molecular changes (Doted circle in (Fig. 2a), and the generation of dendrite and spine remodeling. (2c) Schematic illustration showing some of the changes in dendrite formations (dotted dendrite spines) following learning (synaptic re-wiring) and memory formation (synaptic stabilization). (2d) Schematic illustration showing axonal mechanism of neuroplasticity and repair (rerouting and sprouting) following brain injury. Abbreviations: LTP, long-term potential.

fect LTP in the DG and that the administration of $\mathrm{Na}+$ induces a slow response similar to the acetylcholine and dopamine response, which can be blocked by inhibitors of protein synthesis, increasing the modulation and formation of new synapses. Neurotransmitters like serotonin or histamine have also been proven to have a modulatory effect in LTP. Also, the activation of glutaminergic fibers of cortical origin (facilitated by subcortical fibers) can induce NG in the hippocampus.

The existence of non-functional synapses (so-called silent synapses) has been identified in fish and mammalian species (Atwood, 1999). These silent synapses represent a functional reserve, which is very important in expressing the NP phenomenon and become active by expressing functional AMPA receptor and LTP repetitive activation. Hence, LTP can be considered as a mechanism of synaptic maturity not only during embryogenesis but also in the adult stage. Research conducted by Takumi et al. (1999) have estimated that the adult hippocampal region CA1 consists of at least $25 \%$ of silent synapses and the co-expression of NMDA and AMPA receptors are related to an increase in the postsynaptic density leading to functional changes. The existence of silent synapses and its maturation has also been demonstrated in the mammalian cerebral cortex. It has been shown that postsynaptic density protein-95 (PSD-95) modulate the maturation of silent glutamatergic synapses in principal neurons. Sufficient to govern the duration of the critical period for dominance plasticity in the visual cortex of mice (Huang et al., 2015). Therefore, the formation of new synapses and their continuous modifications (from subtle changes in the synaptic efficiency to the activation of silent synapses) are supported by common molecular mechanisms across the cortical and subcortical regions of the brain (Fig. 2b).

\subsection{Axonal regeneration and plasticity}

It is a well-known fact that axons from the PNS can regenerate from their proximal stem. This process, more elaborated in more primitive vertebrate models (Alunni and Bally-Cuif, 2016) compared to mammals, is limited in the mammalian CNS. However, abortive processes of regeneration among damaged neurons can be seen in mammals, suggesting that central glial cells could limit such axonal regeneration mechanisms (Yiu and He, 2006). Axonal regeneration is particularly useful in long fiber tracts, employing a rewiring mechanism where a neuron fills-up the synaptic communication gap left by dead neurons (rerouting), observed in WM structures as the optical nerve or portion of the spinal cord 
(SC). Other mechanisms, such as collateral sprouting (pruning), occurs when the growth of new processes occurs from healthy axons in neurons from non-affected areas. This creates collateral branches reaching healthy distant regions away from the lesion. Several factors have been postulated that stimulate the collateralization processes, such as the specialization of unaffected postsynaptic spaces, the absence of competitive inhibition, changes in the synaptic activity, presence of degenerative terminals, and the secretion of trophic factors by glial cells, among others.

In terms of molecular biomarkers, the activation of N-methylD-aspartate (NMDA) receptors in postsynaptic neurons and the role of the phosphoprotein GAP-43 (grow associated protein) are related to axon terminal and found in high levels across elongated axons and neurons during collateralization events. Proteins involved in these mechanisms, such as SNAP-25, have also been associated with the axonal and dendritic growth in hippocampal and motoneuron plasticity, and gene expression of Synapsin I is activated at the synaptic contact moment. Overall, collateralization mechanisms can help maintain the axonal and dendritic population and contribute to the excitatory and inhibitory equilibrium and the partial restoration of neural function. These neurobiological phenomena have been extensively described as part of potential recuperative mechanisms associated with memory loss due to neuronal damage. Thus, the manipulation of collateralization processes, followed by the formation of new synaptic contacts, could theoretically play a significant role in recovering NDDs lost functions.

Axonal proliferation processes are typically associated with forming new synapses, redeploying the destroyed functional connections in reactive synaptogenesis. This process is a continuum, starting from the collateralization and concluding with new functional contact points. In this biological model, the presynaptic elements are derived from collateral axons developed after denervation. Ultimately, these microstructural elements evolve from similar small synaptic processes (formed during the embryonic stages) to the synaptic elements observed in adult stages. In the end, postsynaptic processes are modulated by the proliferation of dendrites, which actively participate in the reconstruction of impaired neuronal connectivity. Ultimately, it has been established that dendrites show two different types of response to the lesion; a) the development of specialized regions of presynaptic dendrites and growing of specialized dendrites to develop a dendritic-dendritic communication or $b$ ) a process of gradual atrophy leading to a low density of innervation based on surviving axons (Fig. 2c and 2d). Both phenomena lead to a fair functional recovery without collateralization and an inter-dependent active modulation of the synthesis of proteins.

\subsection{Glia cell plasticity}

Glia cells comprise various cell subtypes, being astrocytes the most abundant cell population (Verkhratsky et al., 2012). Recently, they have been shown to have increasing importance in neuroplasticity and NDDs (Cragnolini et al., 2020). In health and disease, glial cells are far more active than was previously thought and considered powerful synaptic controllers (Van Den Bosch and Robberecht, 2008). Thus, a significant aspect of the initiation and development of neuronal collateralization processes are neuralglial interactions (Stassart et al., 2018). Glial cells are essential for the elimination of degenerated axon terminals. In NDDs, a sequence of glial activation exists, first involving local microglial cells, and subsequently, the astrocyte population. In this coordinated glial interplay, microglia and astrocytes are responsible for producing trophic factors, modulating the axonal regeneration process. In this pathological environment, astrocyte increases the expression of the Tyrosine kinase B (TrkB) receptor, acting as a link with neurotrophic factors for the growing axons. Cellular adhesion molecules such as the neural cell adhesion molecule (NCAM), also contribute to NP events creating biochemical elongation "highways" and participating in the guidance of growing axons. The role of astrocytes is related to network modulation, memory formation, and post-injury repair. Enhancing synaptic plasticity, astrocytes play a key part in mediating memory loss in the hippocampus regulating synaptic transmission and forming new memories. These events may be possible by regulating postsynaptic glutamate receptors (particularly AMPA receptors) dependent on signaling by ATP synthesized in astrocytes (Jo et al., 2014). Thus, the regulations of LTPs (and functional synaptic plasticity) enhances the survivability and functionality of newly formed connections (Ota et al., 2013).

Myelination of the mammalian CNS occurs in a stereotyped and progressive pattern, continuing into adulthood and regulated by oligodendrocyte cells (Purger et al., 2016). Because myelin has dramatic functional consequences for how neurons transmit their signals, myelination has a key role in modulating the brain's network activity (Fields, 2008). Experimental preclinical and human evidence suggests that myelination is sensitive to experiences during development and adulthood. Such variable levels of neuronal activity may be part of a tight experience-dependent change with myelin-forming cells, supporting growing evidence that environmental experiences dynamically control myelin from development to the adult life (Liu et al., 2012). In that regard, several cellular, molecular, and epigenetic mechanisms have been investigated as contributors to myelin plasticity. It appears that the extent of myelin sheath formation may serve to adapt the brain to external events and promote neurogenesis (Fields, 2008). Thus, activitydependent myelination provides a mechanism for selective alteration of the neuronal signal conduction velocity between specific regions of the mammalian cortex and a subsequent change in neural circuit function (Monje, 2018). While the extent to this mechanism remains to be elucidated, early evidence in small mammals shows that premotor circuit activity induces activity-dependent and unequal changes in oligodendrocyte precursor cell (OPC) proliferation and differentiation, exclusively in transcallosal projections (Gibson et al., 2014). Such regulation of the myelination of these specific axons (but not others) could suggest that myelin plasticity is connected to synaptic plasticity as integrated striatal mechanisms to adapt the persistent storage of information (Mensch et al., 2015).

\section{Gray matter neuroplasticity}

Molecular modifications are unmistakably linked to microstructural and functional changes. As an example, new evidence has suggested that in later phases (hours), new detectable synaptic morphological changes can occur after an LTP event, observing an increase in the synaptic density at the local level with 
the formation of areas with an increasing number of local dendritic spines. Thus, the increase of short and thick dendritic spines seems to be temporally posterior to the LTP phenomena. Also, adhesion molecules such as the neural cell adhesion molecule (NCAM) (knowing to be related to the guidance of axonal growth) or presynaptic proteins, like the synaptosome-associated protein 25 kiloDaltons (SNAP-25) are part of an intense protein synthesis mechanism related to LTP, reinforcing the idea of the link between LTP and the differentiation and proliferation responses to NG stimuli. Hence, this supports the evidence of a tight relationship between molecular (LTP) and the ultrastructural GM changes.

\subsection{Superficial gray matter}

Mechanisms of cortical plasticity have been extensively documented and demonstrated in several cortical regions (Jiang et al., 2015; Noppeney et al., 2005; Zatorre et al., 2012). In broad terms, cortical neurons can show two organization patters: a) a classic laminar embryological pattern, and b) a functional organization, which develops during the organism's lifespan from received environmental stimulations and everyone's experience. Studies derived from blindness and interrelated changes in the visual cortex have documented the importance of plastic processes and the functionality of this system. A similar adaptative mechanism operates on other cortical somatosensory and motor cortex, auditive cortex, and other associative brain areas. Besides, more specific pathways associated with thalamocortical and intracortical connectivity established during the embryonic stages (functionally immature and probably silent) could become stimulation-maturation dependent by LTP mechanisms.

The activation of metabotropic and NMDA receptors, the influx of $\mathrm{Ca}^{2+}$, the activation of protein kinases, and protooncogene activation factors (followed by the synthesis of functional proteins leading to the long term morphological changes in spinal dendrites and new synapses) constitute some of the functional chains of events associated with the cortical plasticity. These intrinsic dynamic processes, the cortical connections, can be modulated by extrinsic, subcortical afferents. Hence, neuroplastic remodeling can modify the representation of cortical functions. As an example, Silvian cortical regions of the parietal cortex are in a cross-path area of polymodal function with visual, auditory, and somatosensorial regions. After bilateral visual deprivation, the cortical representation in the Silvian cortical region is rewired or "taken" by the afferents from other cortical modalities.

Further, cortical neuronal networks can also show similar mechanisms of plastic synapses mediated by LTP. As such, these plastic mechanisms intervene in the recovery of lost functions (due to damage or degeneration) without producing important modifications in the mapping of other cortical functions. Likewise, researchers corroborated that the re-arrangement of the auditive cortex (by acoustic stimulus) can be significantly potentiated by the electrical stimulation of the magnocellular basal nucleus, providing cholinergic innervation to the cortex (Kilgard, 1998). This indicates that the mechanisms of cortical plasticity can be modulated by subcortical metabotropic signals, reassuring the interconnection and universality of NP mechanisms across different GM brain structures.

In primary cerebral areas, the capabilities of cortical plasticity mainly decline with the aging process. However, these regions retain a certain degree of cortical plasticity, particularly in associative areas. The apparent closure of the cortical NP capabilities has promoted a new concept of a critical period of neurodevelopment, after which the possibility of functional modifications is no longer possible. Nonetheless, such critical periods are not absolute and can be reopened when the NP mechanisms are reactivated. Although still under investigation, it is presumed that the factors that modulate the reestablishment of NP in the cortex could be associated with the postsynaptic glutaminergic receptors.

\subsection{Deep gray matter}

The hippocampus is a complex deep GM structure in the mammalian brain that shows remarkable potential for structural reorganization. Several strategies have been used to link structural changes to hippocampal function. For example, in in vitro studies of murine adult cells, the experimental reduction of newborn (neurogenic) cells was pharmacologically achieved by systemic administration of antimitotic agents (Bruel-Jungerman et al., 2009). Other virus-based strategies have been developed to prevent neurogenesis exclusively in the hippocampal DG at a specific time in adulthood. (Clelland et al., 2009). Immunohistochemical (IHC) procedures to quantify Bromodeoxyuridine (BrdU) labeled newly born cells are usually used in histological preparations as a complementary noninvasive method to unveil the role of adult-generated neurons in hippocampal functions, particularly for the comparison of mature versus new populations of neurons (Chauhan and Gatto, 2010; Kee et al., 2007).

In preclinical models, various conditions that reduce adult neurogenesis in the DG are associated with learning impairments (Drapeau et al., 2003). Conversely, conditions that increased neurogenesis, such as environmental enrichment and physical exercise, also enhance performance on hippocampal-dependent learning tasks (van Praag, 2005). Recent findings further suggest that a correct differentiation and integration of new neurons maybe necessary for the acquisition of new information and the recall of memories consolidated from previously performed tasks (FarioliVecchioli et al., 2008). Alternative theories about the functions of newborn cells in the hippocampus include the possibility of a rapidly changing population of adult-generated neurons, which may provide a temporary substrate for memory storage. Another important aspect of hippocampal cells is their cellular-selective dependence of NTFs. In studies conducted with BDNF knock-out mice by Zagrebelsky et al. (2018), neuronal architecture and spine morphology of pyramidal cortical and hippocampal neurons had a diverse level of responsiveness to BDNF across different hippocampal and cortical neuronal populations within the same brain area. Possibly due to a link between the different degrees of impairment of the TrkB receptor and a significant decrease in dendritic complexity (Zagrebelsky et al., 2018).

How hippocampal memories are transferred to the prefrontal cortex and the specific structural location of long-term storage and retrieval of memories are still not wholly understood, highlighting the need to investigate structural changes in the hippocampus and related brain regions. A possible explanation could be that learning-induced enhancement of neurogenesis in dendritic spines alters the excitability or activity of the circuitry between the hippocampus and prefrontal cortex. These events, in consequence, modify the microstructure of the prefrontal cortex in a way that 
upholds the storage of memory information. Nonetheless, even if newly created neurons are somehow part of a short-term memory storage system, they must eventually outlive their usefulness, perhaps becoming important by a still unknown function (Leuner and Gould, 2010). At large, preexisting neural circuits undergo adjustments in dendritic complexity and number of synapses, forming an entirely novel neural connections through neurogenesis (Lazarov and Hollands, 2016). Synapses and dendrites of mature hippocampal neurons undergo continuous rearrangement, and entirely new neurons are formed throughout life (Krubitzer and Dooley, 2013). Thus, each life stage (from young adulthood, middle age, and senescence) is characterized by varying degrees of hippocampal plasticity sensitive to a wide range of experiences, many of which appear to have substantial cognitive effects throughout the subject lifespan (van Praag, 2005).

\subsection{Other gray matter structures}

The description of the amygdala reflects an important example of the role of NP in deep GM structures. The amygdala (AMG) is a structure in the limbic system related to conditional fear and emotive memory (Phelps and LeDoux, 2005). It has also been proven to be involved in the storage of other memories connected to the hippocampus. Other studies have found that the electric stimulation of the thalamic nuclei produces evoked potentials in the lateral region of the AMG, followed by the formation of LTPs. The stimulation of the AMG critically regulates the maintenance of the late phase of the LTP. Thus, a weak and short duration LTP enhanced by the AMG (glutaminergic fibers) can be transformed from a temporal window to a strong and lasting LTP. Thus, this electrophysiological model explains at the cellular level the amygdalahippocampus relationships between memory and the influence of emotional and motivational factors. Other studies have described the plastic phenomenon LTD in the cerebellum. Such events seem related to motor learning, dependent on how metabotropic receptors can induce morphological changes in cerebellar stellate cells. Besides, LTP mechanisms could also be found in the striatum, in the nucleus accumbens (NMDA receptor-dependent), and the SC.

Overall, the intricated neuroplastic events across GM cortical and subcortical areas of the CNS are still under extensive and growing research. Biochemical, morphological, and signaling molecules characteristics and neural-inducing activity in specific cell populations have been established using structural markers. As an example, the study of genetically modified mouse models expressing endogenous yellow fluorescent protein (YFP) in distinct neuronal groups have improved our insight of the GM microstructure (Fig. 3). Such indicators are the foundation of further in vivo experimental approaches to describe the increased complexity associated with NP in physiologically intact preclinical models (most commonly used biomarkers are summarized in Table S1).

While the role of neurogenesis in the adult human brain is still subject to intense debate, the study of cortical plasticity opens a new window for the planning of endogenous regenerative strategies aimed to reverse the neuronal damage caused by NDDs (Curtis et al., 2011). A growing number of studies are focused on nonpharmacological interventions to enhance human neuroplasticity in early NDDs stages. As new investigations in the NP field indicated that neuroplastic changes could continue until late in life, this is a tremendously relevant fact due to the high prevalence of NDDs in the aged patient population (Porto et al., 2015). An additional point to consider is also the dynamic nature of NP, indicating that these mechanisms are extremely variable across different individuals and throughout their life experiences (Voss et al., 2017).

In the clinical arena, GM macro and microstructural alterations beforementioned are the biological bases of psychological handicaps, one of the significant burdens in NDDs (Shany-Ur and Rankin, , 2011). Hence, clinical imaging and necessary, preclinical studies can be designed to understand how NDDs change cognitive performance and potentially design strategies to slow-down the progress of the disease (Cho et al., 2008; Choi and Twamley, 2013). However, considering the myriad of factors affecting particular information hubs across different GM structures, monitoring and cognitive remediation strategies (CRS) should include diverse techniques (physical, biochemical, and environmental) to effectively maintain cognitive performance.

Also, current approaches to enhance the cognitive capacity include mental training and reminiscence, stimulating and challenging experiences (visual art and music, physical activities), as well as transcranial magnetic stimulation (TMS) (Herholz et al., 2013). In recent small clinical trials, multimodal non-pharmacological interventions to improve cognitive function have shown that personalized patient treatment with in-depth assessments improved up to 90\% improvement in cognitive performance, stability with dementia, or a delay in their decline (Chalfont et al., 2020). In the long run, physical and cognitive training has also shown a boost in neuroprotective molecules and neurogenesis (Damirchi et al., 2018), demonstrating the constant interplay between environmental and most of the molecular mechanisms previously described.

\section{Neuroplasticity in animal models of neurodegenerative diseases (NDDs)}

From the phylogenetic standpoint, NP is part of a learning mechanism existing in most animals. In some invertebrate species, the acquisition of conditional reactions is based on simple neuronal circuits, a fundamental learning mechanism standard on different animal models. For example, invertebrate models such as the Drosophila melanogaster can learn and thrive through molecular neuroplastic mechanisms. Neurobiological research carried out on non-mammalian vertebrates clearly shows that fish, amphibians, and reptiles do renew most neuronal populations throughout their life. For example, vertebrate organisms like the zebrafish (Danio rerio) have developed specific mechanisms to promote architecture and functionality tissue recovery in CNS damaged tissue (Alunni and Bally-Cuif, 2016; Ganz and Brand, 2016). In such group of animals, their neural stem cells sustain a constitutive status of neurogenesis, as well as latent neural progenitors which can be awakened in the context of an injury (Alunni and BallyCuif, 2016) and particular subspecies of the Teleost fish (Infraclass Teleostei), neurogenic cells from several brain regions migrate guided by radial glial fibers to specific target areas to be part of existing neural networks. Although some of the new cells survive this transformation, others are eliminated through apoptotic cell death mechanisms. Altogether, nonsynaptic transmission (volume transmission), neuro-regeneration and apoptosis enable efficient neurologic rehabilitation after brain injuries (Bachy-Rita, 2005; Zupanc, 2008). 
The differences in biological processes across different small mammalian species is also a typical example of the diversity in NP mechanisms (La Rosa and Bonfanti, 2018). Over the last decades, genetically engineered rodents, such as mice (Mus Musculus) and rats (Rattus norvergicus), have become widely available in neuroscientific research. Using genetic manipulations, they constitute useful tools to elucidate mechanisms underlying specific biological effects by knocking (in or out) specific genes suspected to be involved in specific NDDs molecular pathways (Morfini et al., 2013). Due to a relative genetic similarity with humans, the larger mammals have also been used in NP studies, such cats ( $\mathrm{Fe}$ lis catus), dog (Canis lupus), pigs, (Sus scrofa), sheep (Ovis aries), dolphins (Tursiops truncates \& Stenella coeruleoalba), as well as primates like Rhesus (Macaca Resus) and Marmoset monkeys (Callithrix jacchus) among many other animal models (see Table S2). Along with significant genetic variances between different animal studies, each model varies in a vast number of parameters (promoters used, age of the animal, time of disease onset, transgene expression, neurotransmitter content and amount of overexpression, etc.) (Powers, 2016). Yet, additional strategies, (including identical genetic backgrounds) can reduce the variance associated with differences in profiles of gene expression and phenotypes, leaving the experimental analysis to a shorter number of variables and more reproducible results.

Regardless of the versatility and availability of a vast amount of NP animal models, an increased layer of complexity is achieved by adding genetic mutations associated with human NDDs related neuropathological mechanisms. Some of the main limitations in the study of preclinical animal models are; a) the amount of mutated transgeneses is especially variable, depending on the technique used to overexpress or silence specific genes; $b$ ) the lifespan of most animals is markedly short and is characterized by an accelerated succession of developmental stages far from the normal evolution in the human disease. Still, this constitutes an advantage, making it possible to study neuroplastic processes in NDDs in a relatively short timescale. Several animal studies have already illustrated human-like plasticity in response to physical exercise, e.g., voluntary wheel running in mice (Biedermann et al., 2012) and rats (Sumiyoshi et al., 2014). In contrast, other studies are focused on cognitive training in larger animals (Quallo et al., 2009), indicating that most of the plasticity-related mechanisms are somehow conserved among species. Therefore, considering the biological variability across the different genetic backgrounds and associated phenotype expressions, it is critical to understand how important it is to conduct specific and well-planned preclinical studies (Fisher and Bannerman, 2019).

\subsection{Amyotrophic lateral sclerosis}

Motoneuron loss (MN) is a common feature of several neurodegenerative diseases affecting the spinal cord (SC). Amyotrophic lateral sclerosis (ALS) and spinal muscular atrophy (SMA) represent the most common genetic diseases affecting the SC, brainstem motoneurons along with pyramidal neurons located in the motor cortex (Gatto et al., 2018a, 2019). Maintaining the appropriate motor patterns and coordination of limb movements depends on plasticity within these MN networks. During the last decade, invertebrates and vertebrate transgenic models have been developed to recapitulate some of the genetic, anatomical, and func- tional defects observed in human genes (SOD1, TARDBP, FUS, C9ORF72, TNK, and VCP, among others) as part of the current strategy to help our understanding of the pathophysiology of ALS and the development of therapeutic strategies (Gatto et al., 2020a; Gatto and Weissmann, 2020; McGoldrick et al., 2013).

Preclinical studies of NP in the SOD1-G93A mouse model have described early functional MN abnormalities, with a significant increase in volume and density of dendritic processes, taking place across surviving SC motoneurons to sustain motor performance (Giusto et al., 2020). Another example of SC plasticity in ALS is the ability of cats to recover the capacity to step with their hind legs following an SC transection. For instance, in young feline animals, this ability returns spontaneously, while regular training is required for adult cats to accelerate these mechanisms (de Leon et al., 1998). NP mechanisms have also been studied in neurotoxic rodent models of spinal MN disease (Gulino, 2016), where specific neurotoxins were used to induce selective depletion of spinal MN (cholera toxin B), finding plastic changes in the surviving motoneurons. Thus, the manipulation of these surviving MNs could potentially produce a functional restoration, stimulating these MN compensatory mechanisms (Gulino et al., 2019).

\subsection{Huntington's disease}

Huntington's disease (HD) is an autosomal genetic disease characterized by progressive involuntary choreatic movements, bradykinesia, cognitive decline, and psychiatric syndromes (Finkbeiner, 2011). The aggregation of the mutated huntingtin protein results in neuronal damage of medium spiny neurons of the neostriatum and other neurons in the cortex (Vonsattel, 2007). Migration of neuroblasts from the subventricular zone (SVZ) to the lesion site was observed in neurons expressing neuronal markers (neuronal nuclear antigen (NeuN) and MAP2) in rodent experimental setups of HD (quinolinic lesion model) (Tattersfield et al., 2004). Another widely used group of transgenic mice models (R6/1 and R6/2 lines) includes knock-in transgenic mice with the exon 1 of the human HD gene carrying highly expanded CAG repeats. Additional HD murine research models were developed by the incorporation of the full-length human mutant huntingtin mouse with expanded glutamine repeats on a bacterial (BACHD mice) (Gray et al., 2008) and yeast artificial chromosome (YAC mice) (Hodgson et al., 1999). At the R6 mice hippocampus's dental gyrus (DG), researchers have documented a decline in cell proliferation (Lazic et al., 2006). Specifically, by a reduction of newly generated neurons, without a significant compromise of neuronal differentiation.

Conversely, physical activity and environmental enrichment positively affected the neuronal huntingtin intranuclear inclusion load, leading to further cognitive performance (Pang et al., 2006). Different studies in transgenic rat models also decreased hippocampal progenitor cells, accompanied by an expansion of the quiescent stem cell pool (von Horsten et al., 2003). The migration of neural precursors from the caudate nucleus $(\mathrm{CN})$ to the degenerating striatum was also reported for R6/2 mice (Phillips, 2005). These newly recruited striatal neurons were expressing striatal matured cellular markers like dopamine and cAMP-regulated neuronal phosphoprotein (DARPP-32) and glutamate decarboxylase (GAD). Still, neuroblasts were not able to survive and mature into functional striatal neurons, indicating how the maintenance of a 
proper microenvironment provides a fundamental stimulus for NG and functional integration. Furthermore, experiments locally expanding the expression of BDNF showed that new striate-pallidal projection neurons could survive and be integrated as GABAergic striatal neurons, leading to substantial motor phenotype improvement and prolonged animal survival (Cho et al., 2007).

\subsection{Parkinson's disease}

The loss of dopaminergic neurons in the substantia nigra (SN) of the midbrain and the reduction of neurotransmitters in other brain regions are some of the most relevant neuropathological hallmarks of Parkinson's disease (PD). Among the multiple genetic mutations that generate $\mathrm{PD}$, the most important is $\alpha$-synuclein, which is physiologically found in presynaptic terminals (Masliah et al., 2000). Thus, the accumulation of misfolded $\alpha$-synuclein forms intracellular inclusions in neurons such as Lewy bodies and Lewy neurites. PD toxin-induced models, which have been described for the 1-methyl-4-phenyl1,2,3,6-tetrahydropyridine (MPTP) (Yamada et al., 2004) and 6hydroxydopamine. (6-OHDA) models showing an increase in dopaminergic neurogenesis, reproducing some of the human findings on the olfactory bulbar region (OB) (Winner et al., 2006). In the study of adult neurogenesis, genetically modified PD mice models are under a platelet-derived growth factor (PDGF) promoter (Masliah et al., 2000), as well as a conditional genetic expression by a calcium/calmodulin-dependent protein kinase II alpha promoter (Nuber et al., 2008). Other animal models are based on various $\alpha$-synuclein point mutations (A53T, A30P, E46K) related to rare early $\mathrm{PD}$ onset. Yet, transgenic mice overexpressing of $\alpha$-synuclein reproduce only limited aspects of PD, particularly without the expression of Lewy bodies. Such mutations harm adult neurogenesis in the DG and SVZ brain regions, decreasing neuroblasts and newly generated neurons (Winner et al., 2004). In the conditional calcium/calmodulin-dependent protein kinase II alpha model, there is an association between decreased hippocampal neurogenesis and memory retention (Nuber et al., 2008). Additional roles of glia cell NP has also been described by Udovin et al. (2020), where the Wnt-1 receptor (pathway originally involved in nearby cell-cell communication) also regulates several NP mechanisms (including neuron survival, synapse plasticity, and neurogenesis) favoring neuron survival and viability (Udovin et al., 2020).

\subsection{Alzheimer's disease}

The key features of Alzheimer's disease (AD) include memory impairment, cognitive decline, and olfactory deficits. Pathological findings include; neuronal and synaptic loss, neurofibrillary tangles (due to hyperphosphorylated tau proteins), and deposition of amyloid-beta protein, as well as senile plaques in the basal forebrain cholinergic neurons as well as in the cortex, hippocampus, and amygdala regions, among others (Hardy, 2002). A knock-in mouse model of great interest in AD research is the triple transgenic model, which overexpresses the Swedish mutant APP and mutant P301L Tau protein and the mutant of presenilin 1 (3xTG $\mathrm{AD}$ mice). These mice develop hippocampal tangle-like pathology, neurological deficits, and Ab deposition (Crews et al., 2010). Other common AD murine model is the 5xFAD transgenic mice, which express three mutations in the APP gene (K670N/M671L, I716V, and V717I) and two mutations in the PS1 gene (M146L and L286V). This model induces an aggressive amyloid pathology and heavy neuronal loss. However, when studies compared cognitive performance and adult neurogenesis between those mice models, results seem quite variable (Creighton et al., 2019; Winner et al., 2011). The compromise of adult neurogenesis (preceding neuronal loss) is a common finding across different preclinical AD studies; dysfunctional neurogenesis (decreased and increased) has been extensively reported (Lazarov and Marr, 2010). In addition to newly generated neurons, longer dendrites, increased spine density, and functional responses in early and late stages of the diseases have recently been part of extensive neuropathological reports (Sun et al., 2009). Taken together, such neuronal dysfunctions are part of a larger picture of diverse alterations in synaptic plasticity, spine morphology, and axonal pathology in the context of (physical and social) environmental factors. Globally, these factors have a profound influence on the evolution of the disease and the potential role of NP as a tool for neurological recovery (van Praag, 2005).

\subsection{Other neurodegenerative diseases}

Deficits in synaptic plasticity have been evaluated in other NDDs involving the spinocerebellar function (Rinaldo and Hansel, 2010). In this scenario, identifying mutations related to several hereditary ataxias has led to developing a growing number of valuable animal models (Manto and Marmolino, 2009). As an example, the study of NP in models of spinocerebellar ataxia (SCA) type 1 (SCA1) concluded that external factors (like regular treadmill exercise) changed vital regulatory pathways in the structural and functional plasticity of the neuron, play a critical role in the viable support of SCA1 mice cerebellar neurons (Chuang et al., 2019). More important, (due to overlapping mechanisms across different NDDs) such mutations could have common molecular pathways by genetically conserved NP mechanisms, amenable to be potentially repaired using the proper molecular tools.

\section{Diffusion MRI and neuroplasticity in NDDs.}

With the advent of magnetic resonance imaging (MRI) systems designed to extract functional, structural, and metabolic information of the brain (in a non-invasive manner), preclinical research neuroscience has expanded significantly. In this section, we explore general concepts of diffusion MRI (dMRI) and their application of new imaging techniques in the study of NP in animal models and clinical cohorts of NDDs.

\subsection{General concepts in diffusion MRI}

MRI is founded upon the principles of interaction between an applied magnetic field and an element that possesses spin and charge. The magnetic field is usually represented as a vector, meaning that it has both a magnitude and a direction proportional to the current applied to the system. Short radio frequency (RF) energy pulses (in a narrow bandwidth range) are centered around a central frequency and applied to the tissue sample. During the pulse, the protons absorb a portion of this energy, inducing an intrinsic spin angular momentum. Thus, when the RF transmitter is turned off, the protons immediately start to realign and return to their original orientation from their original equilibrium, emitting the excess of energy at a specific amplitude, frequency, and phase, which is related to the strength of the initially applied magnetic field. 


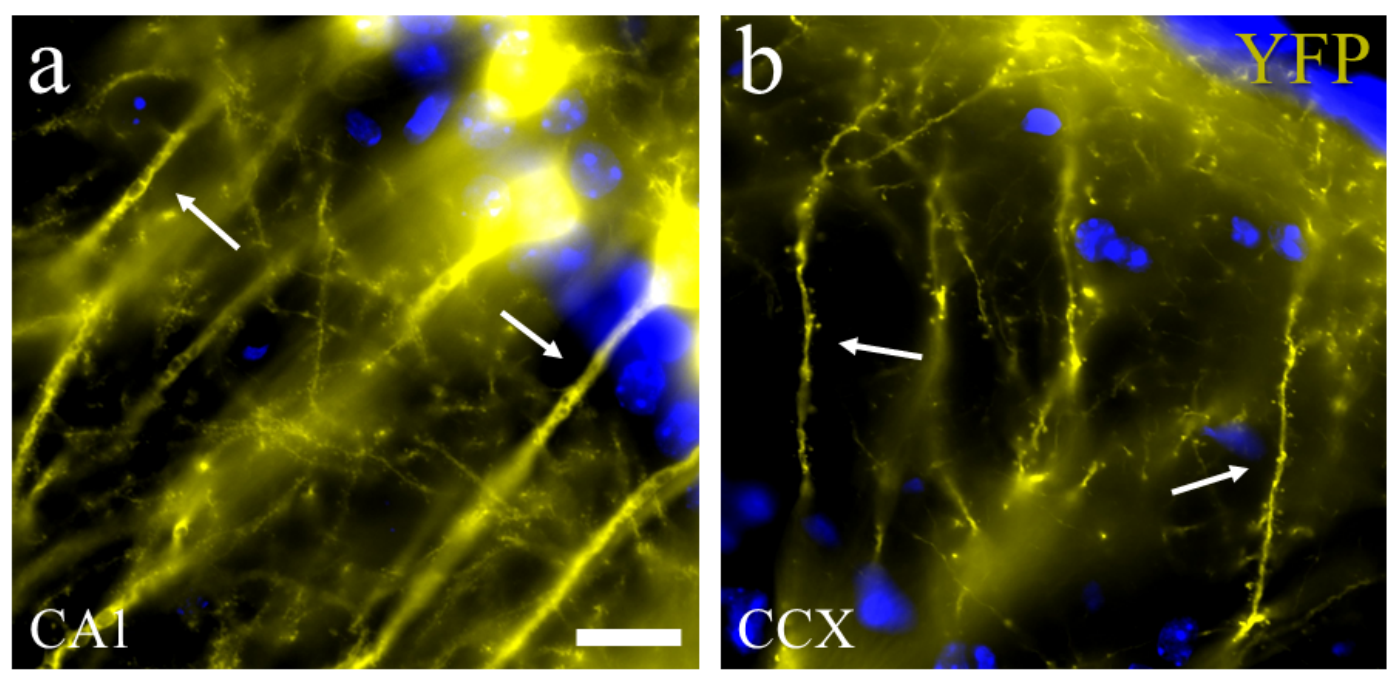

Fig. 3. Neuronal dendrite and spine details from an endogenous fluorescent mice reporter. (3a) Morphological detail of apical dendrite from pyramidal cells of the Cornus Amonus 1 (CA1) hippocampal region in a mouse expressing endogenous yellow fluorescent proteins (YFP) (see arrow). (3b) Histological section showing morphological details from apical dendrites and spines located in the prefrontal cortex of YFP mice (arrows). Scale bar = 5 microns. Abbreviations: YFP, yellow fluorescent protein; CA1, Corpus Amonus region 1 from hippocampus; CCX, cortex.

Additional mathematical processing can map the signals and obtain the scanned object (Dale et al., 2015). Hydrogen nuclei (consisting of a single proton) is a natural choice for analytical MRI techniques in biological samples, due to its abundance in water and fat components. Thus, MRI provides a unique opportunity to non-invasively quantify the diffusional characteristics of a wide range of macro and microstructural processes in the context of different neurological diseases (Gao et al., 2020; Gatto and Weissmann, 2020; Gatto et al., 2015; Gatto and Weissmann, 2019). Then, a probabilistic model to describe the motion of proton particles undergoing diffusion can be built, quantifying the fraction of particles that will move up to a certain distance within a time frame and creating a likelihood that can describe a particular diffusivity behavior based on the particle's displacement. In free diffusion, the distribution of such displacements is theoretically represented by a Gaussian function whose width is determined by the diffusion coefficient. However, in inhomogeneous media (highly heterogenous biological tissues), non-Gaussian models could provide a more archetypal function of the real complexity of biological systems, as explained in the later sections.

One of the first diffusion-weighted MRI (DW-MRI) studies in the neurobiological field was intended to explore the ex vivo microstructure of the unmyelinated giant axons of the squid, where diffusivity anisotropy was intrinsically linked to the axonal neurofilament (cytoskeleton) structure (Beaulieu and Allen, 1994b). Further follow-up work in unmyelinated and myelinated axons of the garfish, using an inhibitor of the microtubule polymerization (Vinblastine), demonstrated that longitudinally oriented barriers to diffusion (axonal membrane or neurofilaments) were shown to be sufficient to generate diffusional anisotropy. Such pioneer neurobiological studies were able to test the ability of dMRI to evaluate the biological nature of the imaging signals (Beaulieu and Allen, 1994a). As such, dMRI is a powerful tool to investigate the intact physiological mechanism and understand the microstructural NP changes (Hamaide et al., 2016). Overall, the combination of dif- ferent MRI modalities allows each voxel to contain an abundance of biological and clinical information. The temporal evolution of functional, structural, and biochemical modifications related to NP can be measured with high precision during the progression of NDDs (Martin et al., 2017).

Even so, an intriguing question is how microstructural variations captured by dMRI may benefit our understanding of NDDs? To answer this query, studies have assessed how changes in neuroimaging correlate with a mono-exponential diffusion model's fitting (Fig. 4). Diffusion tensor imaging (DTI) uses the dMRI Stejskal-Tanner sequence and multidimensional vector algorithms based on six or more gradient directions (Basser and Pierpaoli, 1996; Kingsley, 2006), enough to compute the diffusion tensor to derive neural tract directional information (Koerte and Muehlmann, 2014). The diffusion approach is a relatively simple model of the diffusion process but assumes a homogeneous and linear diffusion regime within each image voxel (Gaussian diffusion). As the diffusion tensor is a symmetric $3 \times 3$ matrix, it can be described by its eigenvalues $\left(\lambda_{1}, \lambda_{2}, \lambda_{3}\right)$ and associated eigenvectors $\left(\mathrm{e}_{1}, \mathrm{e}_{2}, \mathrm{e}_{3}\right)$. At each voxel, the eigenvalues represent the magnitude of diffusion, and the corresponding eigenvectors reflect the maximal and minimal diffusivity directions (Soares et al., 2013). Thus, eigenvalues and eigenvectors are used to process scalar indices such as the axial $(A \times D)$, radial $(\operatorname{Rad}-\mathrm{D})$ as well as mean diffusivity (MD) described as:

$$
\begin{gathered}
\mathrm{A} \times \mathrm{D}=\left(\lambda_{1}\right) \\
\operatorname{Rad}-\mathrm{D}=\left(\lambda_{2}+\lambda_{3}\right) / 2 \\
\mathrm{MD}=\left(\lambda_{1}+\lambda_{2}+\lambda_{3}\right) / 3
\end{gathered}
$$


Overall, $A \times D$ refers to the magnitude of diffusion parallel to fiber tracts, and Rad-D refers to the magnitude of diffusion perpendicular to fiber tracts. Mean diffusivity is the overall directionally averaged magnitude of diffusion. An additional index named fractional anisotropy (FA) has been extensively used to evaluate tissue microstructure (Eqn. 4):

$$
\mathrm{FA}=\sqrt{\frac{1}{2}} \frac{\sqrt{\left(\lambda_{1}-\lambda_{2}\right)^{2}+\left(\lambda_{2}-\lambda_{3}\right)^{2}+\left(\lambda_{1}-\lambda_{3}\right)^{2}}}{\sqrt{\lambda_{1}^{2}+\lambda_{2}^{2}+\lambda_{3}^{2}}}
$$
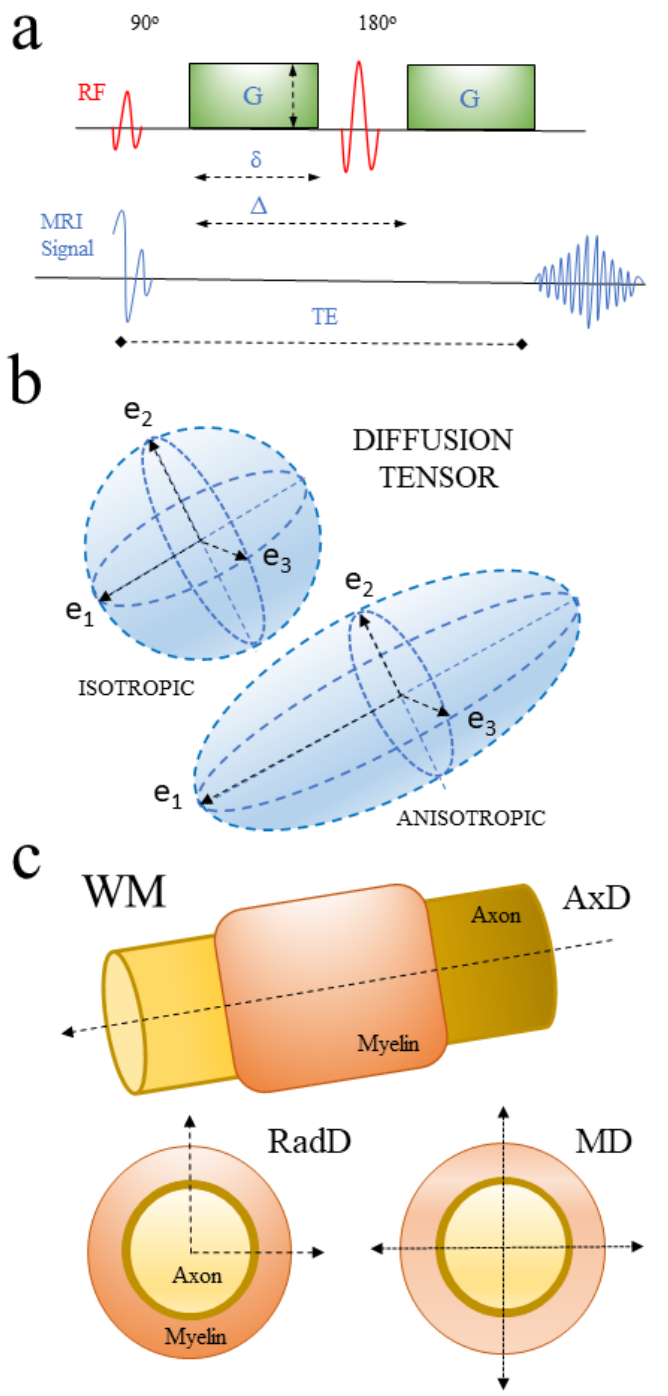

Fig. 4. Diagram showing principles of MR diffusion and diffusion tensor and its association with neural tissue microstructure. (4a) Example of the diffusion MRI Stejskal-Tanner sequence used to generate diffusion tensor imaging (DTI) technique. (4b) Scheme of isotropic and anisotropic diffusion tensors derived. (4c) Diagram showing different DTI parameters and how they relate to white matter structures. Abbreviations: G, gradient pulse magnitude; $\Delta$ gradient pulse time interval; $\delta$, gradient pulse duration; RF, radiofrequency pulse; TE, echo time; e, eigenvalue; WM, white matter; AD, Axial diffusion; RD, Radial diffusion; MD, Mean diffusion.
Additional in vivo basic studies conducted by Song et al. (2003) have shown that each DTI derivative parameters may evaluate specific tissue structures within a voxel. Some of them are related to changes in axonal structure or possibly to structures part of myelinproducing cells. Despite these incredible capabilities, the possibility of DTI to dissect NP-related events at the sub voxel level is substantially limited due to: a) the significantly lower resolution compared with standard optical imaging techniques, b) the assumption of homogenous diffusivity along the voxel, and c) the limited histological co-registration and neuropathological validation (Gatto et al., 2018), all of them still a topic for further investigations and development (Gatto et al., 2020a). Although the use of DTI has been growing exponentially in the neuroscience field (Mori and Zhang, 2006), the interpretations of each diffusivity indices and their biological significance should be held with extreme caution due to a significant amount of limitations. Therefore, the careful consideration of such pitfalls will avoid misconceptions of the dMRI capabilities (Jones et al., 2013).

Additionally, DTI can infer the WM distribution within CNS structures (using a data analysis technique so-called tractography), trying to unveil brain connectivity patterns across different cortical and subcortical regions (Mori et al., 1999). In that regard, DTI needs to include a significant amount of assumptions, leading to several pitfalls when it is used to compare biological data (Calamante, 2019). Hence, the anatomical accuracy of brain connections derived from this technique is also inherently limited. In experimental comparisons between axonal connections, maps derived from neuro-tracer and tractography fiber reconstructions from ex vivo diffusion-weighted imaging (DWI) in datasets obtained from primates (macaque brains) showed an inadequate level of specificity and sensitivity. These disparities were significantly dependent across diverse tractography algorithms (Thomas et al., 2014). Besides, limitations due to the high complexity of WM geometry in the sub voxel regions (Schilling et al., 2017) still lack a comprehensive anatomical connectivity validation in humans.

\subsection{Diffusion MRI and neuroplasticity in NDDs}

dMRI has been a valuable imaging technique to study the neurological progress of patients after rehabilitation treatment. DTI imaging markers (FA and MD) showed lower and higher values, respectively, in deep WM brain structures after physiotherapy in multiple sclerosis (MS) patients using a Siemens Magnetom Trio 3T MR scanner with 20 diffusion directions, 2 b-values $(0,1000$ $\mathrm{s} / \mathrm{mm}^{2}$ ) and a voxel size of $1 \times 1 \times 1 \mathrm{~mm}^{3}$ using a manual segmentation approach (Ibrahim et al., 2011). Moreover, AD studies combining volumetric and DTI measurements in bilateral SVZ regions indicated that MD values increased continuously between controls and different $\mathrm{AD}$ stages. They used 12 diffusion orientations for the diffusion-sensitizing gradients with a b-value of $1000 \mathrm{~s} / \mathrm{mm}^{2}$ (voxel size $1.5 \times 1.5 \times 2.0 \mathrm{~mm}^{3}$ ) by a Siemen 3T Allegra MR imager. These findings highlight the utility of DTI to detect the progressive disruption of tissue caused by the disease, particularly in GM areas well-known related to NP (Cherubini et al., 2010). Other AD clinical studies explored the protective role of lifestyle modifiers, such as physical activity (PA) and its effect on AD-related neurodegeneration of WM tracts. Using a General Electric Signa Excite 3.0 T short bore scanner, DTI was acquired with 25 encoding directions, $\mathrm{b}=0$, and $1000 \mathrm{~s} / \mathrm{mm}^{2}$. It showed a significant 
FA increase in lower-risk groups after high PA (Smith et al., 2016). However, (and contrary to expected) RadD was lower in the lowerrisk group than the observed in high-risk + PA group, implying that PA could exacerbate the APOE- $\epsilon 4$ related processes in neurodegeneration. Thus, the disparities among hardware and clinical imaging protocols across medical institutions could be one of many limiting factors accounting for different results and the challenging interpretation of dMRI data. Moreover, multiple unattainable confounding variables from larger and genetically mixed patient populations datasets could explain these conflictive clinical results. Hence, it could be possible that the deconstruction of such contradictive results will require a more methodic approach and the exploration of more controllable variables by preclinical models.

With further development and applications of ultra-high field dMRI systems (Gatto et al., 2020a), DTI has been the upfront imaging technique to study microstructural changes associated with NP mechanisms across different experimental animal models. For example, this imaging technique has been applied to investigate structural neuroplasticity and gender differences among songbirds. Using a 7T horizontal MR Bruker BioSpin system (with 60 optimally distributed diffusion gradient directions b - 0670 $\mathrm{s} / \mathrm{mm} 2$ and spatial resolutions of $0.06 \times 0.15 \times 0.22 \mathrm{~mm}^{3}$ and $0.07 \times 0.17 \times 0.16 \mathrm{~mm}^{3}$ ), FA maps can distinguish most of the GM and WM-tracts changes connecting major singing nuclei and explore the potential of non-invasive connectomics imaging and visualize the distinct neuronal networks of the sing control system (SCS) (Hamaide et al., 2017). Further, the authors implemented fiber tracking to illustrate the discrimination of all tracts connecting different GM nuclei, as a tool to understand the remarkable plasticity in this brain area (De Groof et al., 2006; Hamaide et al., 2018).

Thus, DTI studies have shown their capability to unveil structural changes associated with different behavioral paradigms and their effects in GM and WM microstructures. Such learning and memory studies performed with a $30-\mathrm{cm}$ bore 7T MRI Bruker scanner (15 noncollinear gradient directions, a single b-value shell at $1000 \mathrm{~s} / \mathrm{mm}^{2}$ and spatial resolution of $0.13 \times 0.13 \times 0.96 \mathrm{~mm}^{3}$ ) by Blumenfeld-Katzir et al. (2011) in rodents also found a significant FA decrease in superficial GM (cortical) related structures, as well as a FA increase in deep WM structures. Moreover, it was found that the Rad-D in certain WM tracts was reduced (which is known to be correlated with myelination status), and such findings were supported by IHC analysis, revealing an increase in myelin basic protein (MBP) staining (Blumenfeld-Katzir et al., 2011).

In dMRI studies, C57BL/6 male mouse models of fear conditioning learning utilizing a horizontal $9.4 \mathrm{~T}$ and $15 \mathrm{~mm}$ coil diameter $\left(b\right.$-value $=1700 \mathrm{~s} / \mathrm{mm}^{2}$ and a voxel size of $0.1 \times 0.1 \times 0.1 \mathrm{~mm}^{3}$, a decrease in FA were located in several superficial (motor and sensory) GM areas (Badea et al., 2019). Besides a detected decrease of FA in deep GM areas (hippocampus), other fear conditioning studies at $7 \mathrm{~T}$ (15 noncollinear gradient directions and a single b-value $=1000 \mathrm{~s} / \mathrm{mm}^{2}$ ) centered in the GM nucleus (amygdala) showed an increase in FA in such a structure (Ding et al., 2013). This demonstrates the diversity of microstructural GM rearrangement in different brain regions and the complexity of circuitry redisposition during NP processes. In a motor learning paradigm conducted by Hofstetter and Assaf (2017) imaging rats (water maze) with a $7 \mathrm{~T}$ Bruker system with a $30 \mathrm{~cm}$ bore (32 gradient directions with a single b-value at $1000 \mathrm{~s} / \mathrm{mm}^{2}$ ), the investigators found a decrease in AxD, Rad-D, and MD diffusivities in several cortical GM and WM regions. In other experimental settings, a finer motor learning paradigm (rotarod) in mice with a Varian multi-channel 7.0T, $40 \mathrm{~cm}$ diameter bore magnet (30 directions with $\mathrm{b}=1917$ $\mathrm{s} / \mathrm{mm}^{2}$, yielding a $130 \mu \mathrm{m}$ isotropic voxel resolution) showed an increase in FA values located in the hippocampus (Scholz et al., 2015), underlying some of the GM microstructural changes occurring in these experimental paradigms. Overall, these results demonstrated the capabilities of dMRI techniques to unveil CNS microstructural changes in specific GM areas associated with NP. Additional details of these DTI studies in preclinical NP models can be found (see (Table. 1).

\subsection{Diffusion MRI: limitations and future directions in neuroplasticity and NDDs}

The right preclinical models (matching to the particular patient's genetic NDD mutation) and the development and application of more accurate imaging tools are vital to optimize and personalize NP therapeutics strategies (Cramer et al., 2011). The collective study of NP and NDDs models can be significantly enhanced by the combination of MRI and histological techniques, offering a unique window of opportunity to study longitudinal changes while controlling for genetic and environmental factors (Tardif et al., 2016).

Although in vivo MRI, animal imaging is an excellent tool to acquire images at high-resolution in physiologically intact mechanisms, it still needs to be biologically validated further to add more solid ground in NP translational research (Gatto et al., 2018). Unfortunately, a limited amount of work has been done to apply this MRI technology to strictly explore potential neuro repair strategies or modulation of neuroplasticity mechanisms in NDDs preclinical models (Table S3). Considering the superior advantage of dMRI to capture microstructural lesions in NDDs, we should initially consider more practical limitations; not all structural lesions in the CNS are followed by a phenotypical expression or clinical signs (silent brain regions). Also, some clinical symptoms are not linked to a unique specific structural lesion detected by dMRI sequences, due to its multiple and diffuse representation (functional redundancy across multiple brain regions). Additional MRI modalities, such as functional MRI (fMRI), have been extensively used to monitor the recovery in neurological diseases (Boyd et al., 2010) and outcomes of multidisciplinary rehabilitation (Péran et al., 2020). as assessed through the most advanced neuroimaging techniques, including diffusion tensor imaging and task-related and resting-state functional magnetic resonance imaging (fMRI). Thus, the combination of fMRI and Magnetic Resonance Spectroscopy (MRS) has been proven a more comprehensive approach to monitor serological neurotransmitter changes combining pharyngeal electrical and TMS in stroke patients with dysphagia (Michou et al., 2015). MRS has been able to also examine neurochemical mechanisms underlying functional reorganization in the primary somatosensory and motor cortices, consequent to upper extremity amputation and transplantation (Jiang et al., 2015). Therefore, these studies stress the importance of integrating structural and functional MRI modalities to characterize the selective changes in cortical neuronal integrity associated with NP. 
Table 1. MRI Studies using Diffusion Tensor Imaging in Animal Models of Neuroplasticity.

\begin{tabular}{|c|c|c|c|c|c|c|c|c|c|c|c|}
\hline $\begin{array}{l}\text { ANIMAL } \\
\text { MODEL }\end{array}$ & COHORT & STUDY & BRAIN STRUCTURE & $\begin{array}{l}\text { GRADIENT } \\
\text { STRENGTH }\end{array}$ & $\begin{array}{c}\text { FIELD } \\
\text { STRENGTH }\end{array}$ & \multicolumn{5}{|c|}{ DTI PARAMETERS } & AUTHORS \\
\hline ADC & FA & AxD & Rad-D & MD & & & & & & & \\
\hline MOUSE & $M(n=24)$ & $\begin{array}{c}\text { Fear } \\
\text { Conditioning }\end{array}$ & $\begin{array}{l}35 \text { GM ROIs (M1, M2, } \\
\text { S1/S2, CPu, V1) }\end{array}$ & $\mathrm{b}=17006 \mathrm{Gdr}$ & $9.4 \mathrm{~T}$ & $\begin{array}{l}\text { (GM) Decreased } \\
\text { (WM) Increased }\end{array}$ & Increased & - & - & - & (Badea et al., 2019) \\
\hline RAT & $\mathrm{M}(\mathrm{n}=113)$ & $\begin{array}{c}\text { Water } \\
\text { MazeTraining }\end{array}$ & 27 GM \& WM ROIs & $\mathrm{b}=1000-$ & $7 \mathrm{~T}$ & - & (NS) & Decreased & Decreased & Decreased & $\begin{array}{l}\text { (Hofstetter and Assaf, } \\
\text { 2017) }\end{array}$ \\
\hline $\begin{array}{l}\text { ZEBRA } \\
\text { FINCH }\end{array}$ & $2 \mathrm{M} / 1 \mathrm{~F}(\mathrm{n}=3)$ & $\begin{array}{l}\text { Structural Sex } \\
\text { differences }\end{array}$ & SCSArea X, HVC, LMAN & $\mathrm{b}=67090 \mathrm{Gd}$ & $\begin{array}{l}9.4 \mathrm{~T}(\text { ex vivo }) \\
7 \mathrm{~T}(\text { in-vivo })\end{array}$ & - & $\mathrm{F}>\mathrm{M}$ & $M>F$ & $M>F$ & $M>F$ & (Hamaide et al., 2017) \\
\hline MOUSE & $M(n=48)$ & Rotarod Test & HPCer, STR, T, HT & $\mathrm{b}=191730 \mathrm{Gd}$ & $7 \mathrm{~T}$ & - & Increased & - & - & - & (Scholz et al., 2015) \\
\hline MOUSE & $M(n=18)$ & $\begin{array}{c}\text { Fear } \\
\text { Conditioning }\end{array}$ & CGAMGHP & $\mathrm{b}=100015 \mathrm{Gd}$ & $7 \mathrm{~T}$ & - & $\begin{array}{c}\text { CG, AMGIn- } \\
\text { creasedHPDecreased }\end{array}$ & $\begin{array}{c}\text { AMG, CG } \\
\text { increasedHP } \\
\text { (NS) }\end{array}$ & (NS) & (NS) & (Ding et al., 2013) \\
\hline RAT & $M(n-68)$ & $\begin{array}{l}\text { Learning and } \\
\text { Memory task }\end{array}$ & $\mathrm{DG}, \mathrm{CG}, \mathrm{PC}, \mathrm{S} 1 / \mathrm{S} 2, \mathrm{CC}$ & $\mathrm{b}=100015 \mathrm{Gd}$ & $7 \mathrm{~T}$ & $\begin{array}{c}\text { (GM) DG, PC, } \\
\text { SGDecreased } \\
(\mathrm{WM}) \mathrm{CC}\end{array}$ & $\begin{array}{l}\text { (GM) CGDecreased } \\
(\mathrm{WM}) \text { CCIncreased }\end{array}$ & - & - & - & $\begin{array}{c}\text { (Blumenfeld-Katzir et } \\
\text { al., 2011) }\end{array}$ \\
\hline RAT & $\mathrm{M}(\mathrm{n}=51)$ & $\begin{array}{l}\text { Epileptic KA } \\
\text { Model }\end{array}$ & DG & $\mathrm{b}=10006 \mathrm{Gd}$ & $\begin{array}{l}9.4 \mathrm{~T}(\text { ex vivo }) \\
7 \mathrm{~T} \text { (in-vivo) }\end{array}$ & - & (GM) Increased & Increased & (NS) & - & (Laitinen et al., 2010) \\
\hline STARLING & $M(n=12)$ & $\begin{array}{c}\text { Seasonal Singing } \\
\text { Patters }\end{array}$ & $\begin{array}{c}\text { SCS (HVC-RA tract, RA, } \\
\text { OM, DM) }\end{array}$ & $-6 \mathrm{Gd}$ & $7 \mathrm{~T}$ & - & (WM) Decreased & Decreases & Increased & (NS) & (De Groof et al., 2008) \\
\hline
\end{tabular}

MRI Studies using Diffusion Tensor Imaging in Animal Models of Neuroplasticity.

Abbreviations: DTI: diffusion tensor imaging; ADC: apparent diffusion coefficient; A x D: axial diffusion; Rad-D: radial diffusion; MD: mean diffusion; M: male; F: female; GM: grey matter; WM: white matter; M1: primary motor cortical area; M2: supplementary motor cortical area; S1: primary cortical sensory area; S2: supplementary cortical sensory area; SCS: song control system; HVC: high vocal center; LMAN: the lateral magnocellular nucleus of the anterior Nigro-pallium; RA: the nucleus of the arcopallium; HP: hippocampus; AMG: amygdala; T: thalamus; HT: hypothalamus; STR: striatum; CG: central gyrus; PC: parietal cortex; CC: corpus callosum; KA: kainic acid; Gd: gradient directions; NS: statistically non-significant; T: Tesla. 

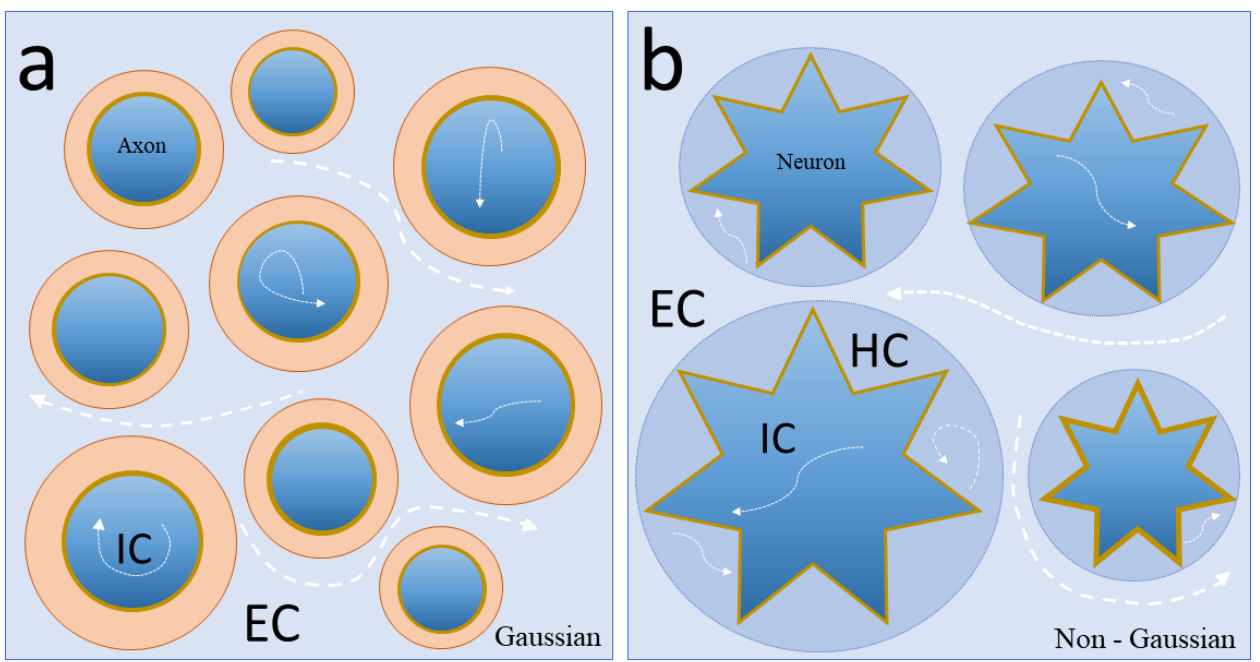

Fig. 5. Schematic diagram of Gaussian and non-Gaussian diffusion MRI techniques applied to different nerve tissue microstructures. (5a) Schematic diagram of a transversal section from a sub-voxel region located in the white matter area. Note that water diffusion (white arrows) In Gaussian noncompartmental models (DTI) is an average of all compartments in the sub-regions (IC + EC). (5b) Transversal section from a sub-voxel region located in the grey matter area. Note that due to increased structural complexity in microstructure, different water diffusion regimes (white arrows) can be explored by non-Gaussian models. Such models can theoretically provide parameters related to individual morphological changes from different nervous tissue (IC, EC, HC). Abbreviations: WM, white matter; GM, grey matter; IC, extracellular compartment; EC, extracellular compartment; HC hindered compartment.

On a more technical viewpoint, due to the current significant disparity between dMRI voxel dimensions and cellular structures, there is a limited appraisal of the profound microcircuit changes within the GM and its architectural alterations (McColgan et al., 2020). The application of current diffusion models in medicine or biology has raised many questions ins the scientific community (Calamante, 2019; Little and Holloway, 2007). As an example, when a significant part of cognitive functions and cortical plasticity events are intrinsically associated with cortical and subcortical GM structures, mono-exponential diffusion tensor models seem to be substantially limited to capture such complexity across a heterogeneous media such as neuronal tissues. To this end, multicompartmental dMRI techniques, such as neurite orientation dispersion imaging (NODDI) (Zhang et al., 2012) or nonGaussian techniques, like diffusion kurtosis imaging (DKI) (Gatto, 2020b), continuous random walk model (CTRW) (Gatto et al., 2019) and fractional-order calculus model (FROC) (Liang et al., 2016) (among other diffusion models), could be considered to explore the hindered sub-compartments linked to the extreme intricacy of CNS microstructure (Fig. 5).

Where the dynamic and adaptative changes in dendrite arborization have been already characterized in neurophysiological NDDs studies (Elbasiouny et al., 2012), the whole network organization fundamentally influences brain disease (Fornito and Bullmore, 2015). Thus, a non-invasive imaging technique grounded in a whole network approach is intuitively essential to understanding early NP responses (Poldrack, 2000; Tardif et al., 2016). In that regard, our previous studies using CTRW dMRI methods found an overall increase in brain tissue complexity (1- $\alpha$ parameter) in animal models of HD at the early stages of the disease. Such changes were not only driven by an increment in glia cell population involved in brain repair processes (astrocytes) measured by fluorescent and IHC microscopy techniques, but also by com- plex adaptative changes in WM axonal structural features such as size, density, and tortuosity (or waviness) measured by electron microscopy methods (Gatto et al., 2019). Thus, early microstructural NP could be related to axonal pruning adaptative mechanisms (Low and Cheng, 2006) without much early neuronal cell death finding (Rattray et al., 2013). Hence, structural network organizations in NDDs can be somehow preserved despite the loss of WM tract integrity, possibly by biologically driven self-adjusted mechanisms (Menke et al., 2016; Mole et al., 2016; Powell et al., 2018). Thus, further combined imaging analysis could hold a more accurate key in the study of the adaptative network mechanism (Aydogan et al., 2018), improving our understanding of the dynamics of the NP phenomenon and design better therapeutic approaches for NDDs.

\section{Concluding remarks}

During neurodevelopment and the adult age, the structural shape of the nervous system is continuously influenced by subtle functional modifications triggered by internal and external factors. The study of molecular and cellular events is an important step to understand the processes governing such microstructural changes as a response to environmental factors, NP imprint repetitive stimulus at the cellular level modifying axonal and dendritic growing processes, as well as the formation of new synaptic structures. Notably, a continuum of genetic and molecular NP mechanisms are well-preserved across different animal species, making them highly valuable for preclinical studies. However, several pitfalls in current dMRI techniques, and it's still lack of fully biological validation imposes several limitations to extract useable and representative information to move this field further. Overall, the proper combination of transgenic preclinical models and novel imaging models and multi-imaging modalities is hugely critical to understanding the NP phenomena in physiological intact biologi- 
cal models. This is a significant step towards designing more efficient therapeutic strategies to ameliorate the devastating effects of NDDs in patients and their families.

\section{Acknowledgments}

I would like to thank Dr. Ariel Finkielsztein for their critical reading of the neurobiological section of this manuscript and Dr. Richard Magin for his suggestions and proofreading of the MRI portion of this manuscript. I want to also recognize Drs. Ariel Finkielsztein and Ronen Sumagin for their help to image the fluorescent mouse brain images presented in this review.

\section{Conflict of Interest}

The authors declare no conflict of interest.

\section{Supplementary material}

Supplementary material associated with this article can be found, in the online version, at https://jin.imrpress.com/EN/ $10.31083 /$ j.jin.2020.03.165.

\section{Submitted: May 26, 2020}

Revised: September 08, 2020

Accepted: September 10, 2020

Published: September 30, 2020

\section{References}

Alunni, A. and Bally-Cuif, L. (2016) A comparative view of regenerative neurogenesis in vertebrates. Development 143, 741-753.

Ashburner, J. and Friston, K. J. (2000) Voxel-based morphometry-the methods. Neuroimage 11, 805-821.

Atwood, H. L. (1999) Silent synapses in neural plasticity: Current evidence. Learning and Memory 6, 542-571.

Aydogan, D. B., Jacobs, R., Dulawa, S., Thompson, S. L., Francois, M. C., Toga, A. W., Dong, H., Knowles, J. A. and Shi, Y. (2018) When tractography meets tracer injections: A systematic study of trends and variation sources of diffusion-based connectivity. Brain Structure and Function 223, 2841-2858.

Bach-y-Rita, P. (2005) Emerging concepts of brain function. Journal of Integrative Neuroscience 4, 183-205.

Badea, A., Ng, K. L., Anderson, R. J., Zhang, J., Miller, M. I. and O'Brien, R. J. (2019) Magnetic resonance imaging of mouse brain networks plasticity following motor learning. PLoS One 14, e0216596.

Bagley, J., LaRocca, G., Jimenez, D. A. and Urban, N. N. (2007) Adult neurogenesis and specific replacement of interneuron subtypes in the mouse main olfactory bulb. BMC Neuroscience 8, 92 .

Bai, Y. and Suzuki, T. (2020) Activity-dependent synaptic plasticity in drosophila melanogaster. Frontiers in Physiology 11, 161.

Bashir, Z. I., Alford, S., Davies, S. N., Randall, A. D. and Collingridge, G. L. (1991) Long-term potentiation of NMDA receptor-mediated synaptic transmission in the hippocampus. Nature 349, 156-158.

Basser, P. J. and Pierpaoli, C. (1996) Microstructural and physiological features of tissues elucidated by quantitative-diffusion-tensor MRI. Journal of Magnetic Resonance, Series B 111, 209-219.

Beaulieu, C. and Allen, P. S. (1994a) Determinants of anisotropic water diffusion in nerves. Magnetic Resonance in Medicine 31, 394-400.

Beaulieu, C. and Allen, P. S. (1994b) Water diffusion in the giant axon of the squid: Implications for diffusion-weighted MRI of the nervous system. Magnetic Resonance in Medicine 32, 579-583.

Bergado-Rosado, J. A. and Almaguer Melián, W. (2000) Mecanismos celulares de la neuroplasticidad. Revista De NeurologÍa 31, 1074. (In Spanish)

Biedermann, S., Fuss, J., Zheng, L., Sartorius, A., Falfán-Melgoza, C., Demirakca, T., Gass, P., Ende, G. and Weber-Fahr, W. (2012) In vivo voxel based morphometry: Detection of increased hippocampal vol- ume and decreased glutamate levels in exercising mice. Neuroimage 61, 1206-1212.

Bijoch, L., Borczyk, M. and Czajkowski, R. (2020) Bases of Jerzy Konorski's theory of synaptic plasticity. European Journal of Neuroscience 51, 1857-1866.

Binder, D. K. and Scharfman, H. E. (2004) Brain-derived neurotrophic factor. Growth Factors 22, 123-131.

Blumenfeld-Katzir, T., Pasternak, O., Dagan, M. and Assaf, Y. (2011) Diffusion MRI of structural brain plasticity induced by a learning and memory task. PLoS One 6, e20678.

Boyd, L. A., Vidoni, E. D. and Wessel, B. D. (2010) Motor learning after stroke: Is skill acquisition a prerequisite for contralesional neuroplastic change? Neuroscience Letters 482, 21-25.

Brown, S. D. and Bottjer, S. W. (1993) Testosterone-induced changes in adult canary brain are reversible. Journal of Neurobiology 24, 627-640.

Bruel-Jungerman, E., Veyrac, A., Dufour, F., Horwood, J., Laroche, S. and Davis, S. (2009) Inhibition of PI3K-Akt signaling blocks exercisemediated enhancement of adult neurogenesis and synaptic plasticity in the dentate gyrus. PLoS One 4, e7901.

Budnik, V. (1996) Synapse maturation and structural plasticity at Drosophila neuromuscular junctions. Current Opinion in Neurobiology 6, 858-867.

Cajal, S. R.y. (2002) Texture of the Nervous System of Man and the Vertebrates. Vol. 3. New York: Springer.

Calamante, F. (2019) The seven deadly sins of measuring brain structural connectivity using diffusion MRI streamlines fibre-tracking. Diagnostics 9, 115.

Cerri, S., Bottiroli, G., Bottone, M. G., Barni, S. and Bernocchi, G. (2009) Cell proliferation and death in the brain of active and hibernating frogs. Journal of Anatomy 215, 124-131.

Chalfont, G., Milligan, C. and Simpson, J. (2020) A mixed methods systematic review of multimodal non-pharmacological interventions to improve cognition for people with dementia. Dementia 19, 1086-1130.

Chauhan, N. B. and Gatto, R. (2010) Synergistic benefits of erythropoietin and simvastatin after traumatic brain injury. Brain Research, $\mathbf{1 3 6 0}$, 177-192.

Cherubini, A., Spoletini, I., Péran, P., Luccichenti, G., Di Paola, M., Sancesario, G., Gianni, W., Giubilei, F., Bossù, P., Sabatini, U., Caltagirone, C. and Spalletta, G. (2010) A multimodal MRI investigation of the subventricular zone in mild cognitive impairment and Alzheimer's disease patients. Neuroscience Letters 469, 214-218.

Cho, H., Yang, D. W., Shon, Y. M., Kim, B. S., Kim, Y. I., Choi, Y. B., Lee, K. S., Shim, Y. S., Yoon, B., Kim, W. and Ahn, K. J. (2008) Abnormal integrity of corticocortical tracts in mild cognitive impairment: A diffusion tensor imaging study. Journal of Korean Medical Science 23, 477 .

Cho, S., Benraiss, A., Chmielnicki, E., Samdani, A., Economides, A. and Goldman, S. A. (2007) Induction of neostriatal neurogenesis slows disease progression in a transgenic murine model of Huntington disease. Journal of Clinical Investigation 117, 2889-2902.

Choi, J. and Twamley, E. W. (2013) Cognitive rehabilitation therapies for Alzheimer's disease: A review of methods to improve treatment engagement and self-efficacy. Neuropsychology Review 23, 48-62.

Chuang, C., Chang, J., Soong, B., Chuang, S., Lin, T., Cheng, W., Orr, H. T. and Liu, C. (2019) Treadmill training increases the motor activity and neuron survival of the cerebellum in a mouse model of spinocerebellar ataxia type 1. The Kaohsiung Journal of Medical Sciences 35, 679-685.

Clelland, C. D., Choi, M., Romberg, C., Clemenson, G. D., Fragniere, A., Tyers, P., Jessberger, S., Saksida, L. M., Barker, R. A., Gage, F. H. and Bussey, T. J. (2009) A functional role for adult hippocampal neurogenesis in spatial pattern separation. Science 325, 210-213.

Cornez, G., Jonckers, E., ter Haar, S. M., Van der Linden, A., Cornil, C. A. and Balthazart, J. (2018) Timing of perineuronal net development in the zebra finch song control system correlates with developmental song learning. Proceedings of the Royal Society B: Biological Sciences 285, 20180849.

Cirillo, G., Cragnolini, A., Lampitella, G., Virtuoso, A., Viscovo, I., Panetsos, F. and Papa, M. (2020) Regional brain susceptibility to neurode- 
generation: What is the role of glial cells? Neural Regeneration Research $\mathbf{1 5}, 838$.

Cragnolini, A. B. L., G., Virtuoso, A., Viscovo, I., Panetsos, F., Papa, M. and Cirillo, G. (2020) Regional brain susceptibility to neurodegeneration: What is the role of glial cells? Neural Regeneration Research $\mathbf{1 5}$, 838-842.

Cramer, S. C., Sur, M., Dobkin, B. H., O'Brien, C., Sanger, T. D., Trojanowski, J. Q., Rumsey, J. M., Hicks, R., Cameron, J., Chen, D., Chen, W. G., Cohen, L. G., de Charms, C., Duffy, C. J., Eden, G. F., Fetz, E. E., Filart, R., Freund, M., Grant, S. J., Haber, S., Kalivas, P. W., Kolb, B., Kramer, A. F., Lynch, M., Mayberg, H. S., McQuillen, P. S., Nitkin, R., Pascual-Leone, A., Reuter-Lorenz, P., Schiff, N., Sharma, A., Shekim, L., Stryker, M., Sullivan, E. V., Vinogradov, S. (2011) Harnessing neuroplasticity for clinical applications. Brain 134, 15911609.

Creighton, S. D., Mendell, A. L., Palmer, D., Kalisch, B. E., MacLusky, N. J., Prado, V. F., Prado, M. A. M. and Winters, B. D. (2019) Dissociable cognitive impairments in two strains of transgenic Alzheimer's disease mice revealed by a battery of object-based tests. Scientific Reports $\mathbf{9}$, 57.

Crews, L., Rockenstein, E. and Masliah, E. (2010) APP transgenic modeling of Alzheimer's disease: Mechanisms of neurodegeneration and aberrant neurogenesis. Brain Structure and Function 214, 111-126.

Curtis, M. A., Kam, M. and Faull, R. L. M. (2011) Neurogenesis in humans. European Journal of Neuroscience 33, 1170-1174.

Dale, B. M. B., Mark A., Semelka, Richard C. and Brown, Mark A. (2015) MRI: Basic principles and applications. Chichester, West Sussex. Hoboken, NJ: John Wiley \& Sons, Ltd.

Damirchi, A., Hosseini, F., Babaei, P. (2018) Mental training enhances cognitive function and BDNF more than either physical or combined training in elderly women with mci: A small-scale study. American Journal of Alzheimer's Disease and other Dementias 33, 20-29.

Dawley, E. M., Fingerlin, A., Hwang, D., John, S. S. and Stankiewicz, C. A. (2000) Seasonal cell proliferation in the chemosensory epithelium and brain of red-backed salamanders, Plethodon cinereus. Brain Behaviour Evolution 56, 1-13.

De Groof, G., Verhoye, M., Van Meir, V., Balthazart, J. and Van der Linden, A. (2008) Seasonal rewiring of the songbird brain: An in vivo MRI study. European Journal of Neuroscience 28, 2475-2485.

De Groof, G., Verhoye, M., Van Meir, V., Tindemans, I., Leemans, A. and Van der Linden, A. (2006) In vivo diffusion tensor imaging (DTI) of brain subdivisions and vocal pathways in songbirds. Neuroimage 29 754-763.

de Leon, R. D., Hodgson, J. A., Roy, R. R., Edgerton, V. R. (1998) Locomotor capacity attributable to step training versus spontaneous recovery after spinalization in adult cats. Journal of Neurophysiology 79 $1329-1340$

Decimo, I., Bifari, F., Krampera, M. and Fumagalli, G. (2012) Neural stem cell niches in health and diseases. Current Pharmaceutical Design 18, $1755-1783$

Delgado-Gonzalez, F. J., Gonzalez-Granero, S., Trujillo-Trujillo, C. M., García-Verdugo, J. M. and Damas-Hernandez, M. C. (2011) Study of adult neurogenesis in the gallotia galloti lizard during different seasons. Brain Research 1390, 50-58.

Dhari, Z., Ishibahi, N., Jonas, R. and Leonetti, C. (2019) Reduced neurogenic activity in the subventricular zone after cardiopulmonary bypass in a juvenile porcine model. Pediatrics 144, 348-348.

Ding, A. Y., Li, Q., Zhou, I. Y., Ma, S. J., Tong, G., McAlonan, G. M. and Wu, E. X. (2013) MR diffusion tensor imaging detects rapid microstructural changes in amygdala and hippocampus following fear conditioning in mice. PLoS One 8, e51704.

Drapeau, E., Mayo, W., Aurousseau, C., Le Moal, M., Piazza, P. and Abrous, D. N. (2003) Spatial memory performances of aged rats in the water maze predict levels of hippocampal neurogenesis. Proceedings of the National Academy of Sciences 100, 14385-14390.

Elbasiouny, S., Quinlan, K., Eissa, T. and Heckman, C. (2012) Electrophysiological abnormalities in SOD1 transgenic models in amyotrophic lateral sclerosis: The commonalities and differences. Amyotrophic Lateral Sclerosis 157-174.
Fagiolini, M., Jensen, C. L. and Champagne, F. A. (2009) Epigenetic influences on brain development and plasticity. Current Opinion in $\mathrm{Neu}$ robiology 19, 207-212.

Farioli-Vecchioli, S., Saraulli, D., Costanzi, M., Pacioni, S., Cinà, I., Aceti, M., Micheli, L., Bacci, A., Cestari, V. and Tirone, F. (2008) The timing of differentiation of adult hippocampal neurons is crucial for spatial memory. Plos Biology 6, e246

Fields, R. D. (2008) Oligodendrocytes changing the rules: Action potentials in glia and oligodendrocytes controlling action potentials. The Neuroscientist 14, 540-543.

Finkbeiner, S. (2011). Huntington's Disease. Cold Spring Harbor Perspectives in Biology 3, a007476.

Fisher, E. M. C. and Bannerman, D. M. (2019) Mouse models of neurodegeneration: Know your question, know your mouse. Science Translational Medicine 11, eaaq1818.

Fornito, A. and Bullmore, E. T. (2015) Connectomics: A new paradigm for understanding brain disease. European Neuropsychopharmacology 25, 733-748.

Ganz, J. and Brand, M. (2016) Adult neurogenesis in fish. Cold Spring Harbor Perspectives in Biology 8, a019018.

Gao, J., Jiang, M., Magin, R. L., Gatto, R. G., Morfini, G., Larson, A. C. and Li, W. (2020) Multicomponent diffusion analysis reveals microstructural alterations in spinal cord of a mouse model of amyotrophic lateral sclerosis ex vivo. PLoS One 15, e0231598.

Garcia, P., Youssef, I., Utvik, J. K., Florent-Béchard, S., Barthélémy, V., Malaplate-Armand, C., Kriem, B., Stenger, C., Koziel, V., Olivier, J. -L., Escanye, M. C., Hanse, M., Allouche, A., Desbene, C., Yen, F. T., Bjerkvig, R., Oster, T., Niclou, S. P. and Pillot, T. (2010) Ciliary neurotrophic factor cell-based delivery prevents synaptic impairment and improves memory in mouse models of Alzheimer's disease. Journal of Neuroscience 30, 7516-7527.

Gatto, R. G., Li, W., Gao, J. and Magin, R. L. (2018a) In vivo diffusion MRI detects early spinal cord axonal pathology in a mouse model of amyotrophic lateral sclerosis. NMR in Biomedicine 31, e3954.

Gatto, R. G., Amin, M., Finkielsztein, A., Weissmann, C., Barrett, T., Lamoutte, C., Uchitel, O., Sumagin, R., Mareci, T. H. and Magin, R. L. (2019) Unveiling early cortical and subcortical neuronal degeneration in ALS mice by ultra-high field diffusion MRI. Amyotrophic Lateral Sclerosis and Frontotemporal Degeneration 20, 549-561.

Gatto, R. G., Weissmann, C., Amin, M., Finkielsztein, A., Sumagin, R., Mareci, T. H., Uchitel, O. D. and Magin, R. L. (2020a) Assessing neuraxial microstructural changes in a transgenic mouse model of early stage Amyotrophic Lateral Sclerosis by ultra $\square$ high field MRI and diffusion tensor metrics. Animal Models and Experimental Medicine 3 , 117-129.

Gatto, R. (2020b). Editorial for "Evaluating the therapeutic effect of low $\square$ intensity transcranial ultrasound on traumatic brain injury with diffusion kurtosis imaging". Journal of Magnetic Resonance Imaging 52, 532-533.

Gatto, R. G. and Weissmann, C. (2020) Neuroimaging on Preclinical Models of ALS. In, Sørensen, J. (eds.) Amyotrophic Lateral Sclerosis: From Diagnosis to Treatment (pp. 1-97). Nova Science Publisher.

Gatto, R., Chauhan, M. and Chauhan, N. (2015) Anti-edema effects of rhEpo in experimental traumatic brain injury. Restorative Neurology and Neuroscience 33, 927-941.

Gatto, R. G., Ye, A. Q., Colon-Perez, L., Mareci, T. H., Lysakowski, A., Price, S. D., Brady, S. T., Karaman, M., Morfini, G. and Magin, R. L. (2019) Detection of axonal degeneration in a mouse model of Huntington's disease: Comparison between diffusion tensor imaging and anomalous diffusion metrics. Magnetic Resonance Materials in Physics, Biology and Medicine 32, 461-471.

Gatto, R. G. and Weissmann, C. (2019) Diffusion tensor imaging in preclinical and human studies of Huntington's disease: What have we learned so far? Current Medical Imaging formerly Current Medical Imaging Reviews 15, 521-542.

Gatto, R. G., Amin, M. Y., Deyoung, D., Hey, M., Mareci, T. H. and Magin, R. L. (2018) Ultra-high field diffusion MRI reveals early axonal pathology in spinal cord of ALS mice. Translational Neurodegeneration 7, 20 
Geribaldi-Doldán, N., Gómez-Oliva, R., Domínguez-García, S., NunezAbades, P. and Castro, C. (2019) Protein kinase C: Targets to regenerate brain injuries? Frontiers in Cell and Developmental Biology7, 39.

Ghosh, S. and Hui, S. P. (2016) Regeneration of zebrafish CNS: Adult neurogenesis. Neural Plasticity 2016, 5815439.

Gibson, E. M., Purger, D., Mount, C. W., Goldstein, A. K., Lin, G. L., Wood, L. S., Inema, I., Miller, S. E., Bieri, G., Zuchero, J. B., Barres, B. A., Woo, P. J., Vogel, H. and Monje, M. (2014) Neuronal activity promotes oligodendrogenesis and adaptive myelination in the mammalian brain. Science 344, 1252304-1252304.

Giusto, E., Codrich, M., Leo, G., Francardo, V., Coradazzi, M., Parenti, R., Gulisano, M., Vicario, N., Gulino, R. and Leanza, G. (2020) Compensatory changes in degenerating spinal motoneurons sustain functional sparing in the SOD1 $\square$ G93A mouse model of amyotrophic lateral sclerosis. Journal of Comparative Neurology 528, 231-243.

Gould, E. (1999) Neurogenesis in adulthood: A possible role in learning. Trends in Cognitive Sciences 3, 186-192.

Gray, M., Shirasaki, D. I., Cepeda, C., Andre, V. M., Wilburn, B., Lu, X., Tao, J., Yamazaki, I., Li, S., Sun, Y. E., Li, X., Levine, M. S. and Yang, X. W. (2008) Full-length human mutant huntingtin with a stable polyglutamine repeat can elicit progressive and selective neuropathogenesis in BACHD mice. Journal of Neuroscience 28, 6182-6195.

Gulino, R. (2016) Neuroplasticity and repair in rodent neurotoxic models of spinal motoneuron disease. Neural Plasticity 2016, 1-11.

Gulino, R., Vicario, N., Giunta, M., Spoto, G., Calabrese, G., Vecchio, M., Gulisano, M., Leanza, G. and Parenti, R. (2019) Neuromuscular plasticity in a mouse neurotoxic model of spinal motoneuronal loss. International Journal of Molecular Sciences 20, 1500.

Gulyaeva, N. V. (2017) Molecular mechanisms of neuroplasticity: An expanding universe. Biochemistry (Moscow) 82, 237-242.

Hamaide, J., Bigler, A. and Van der Linden, A. (2018) MRI: An ideal tool to explore the neural substrate of vocal communication in songbirds. Handbook of Behavioral Neuroscience 28, 457-479.

Hamaide, J., De Groof, G. and Van der Linden, A. (2016) Neuroplasticity and MRI: A perfect match. Neuroimage 131, 13-28.

Hamaide, J., De Groof, G., Van Steenkiste, G., Jeurissen, B., Van Audekerke, J., Naeyaert, M., Van Ruijssevelt, L., Cornil, C., Sijbers, J., Verhoye, M. and Van der Linden, A. (2017) Exploring sex differences in the adult zebra finch brain: In vivo diffusion tensor imaging and ex vivo super-resolution track density imaging. Neuroimage 146, 789-803.

Hardy, J. (2002) The Amyloid hypothesis of Alzheimer's disease: Progress and problems on the road to therapeutics. Science 297, 353-356.

Herholz, S. C., Herholz, R. S. and Herholz, K. (2013) Nonpharmacological interventions and neuroplasticity in early stage Alzheimer's disease. Expert Review of Neurotherapeutics 13, 12351245.

Hodgson, J. G. A., N., Gutekunst, C. A., Leavitt, B. R., LePiane, F., Singaraja, R., Smith, D. J., Bissada, N., McCutcheon, K., Nasir, J., Jamot, L., Li, X. J., Stevens, M. E., Rosemond, E., Roder, J. C., Phillips, A. G., Rubin, E. M., Hersch, S. M. and Hayden, M. R. (1999) A YAC mouse model for Huntington's disease with full-length mutant huntingtin, cytoplasmic toxicity, and selective striatal neurodegeneration. Neuron 23, 181-192.

Hofstetter, S. and Assaf, Y. (2017) The rapid development of structural plasticity through short water maze training: A DTI study. Neuroimage 155, 202-208.

Huang, E. J. and Reichardt, L. F. (2001) Neurotrophins: Roles in neuronal development and function. Annual Review of Neuroscience 24, 677736.

Huang, X., Stodieck, S. K., Goetze, B., Cui, L., Wong, M. H., Wenzel, C., Hosang, L., Dong, Y., Löwel, S. and Schlüter, O. M. (2015) Progressive maturation of silent synapses governs the duration of a critical period. Proceedings of the National Academy of Sciences 112, E3131-E3140.

Ibrahim, I., Tintera, J., Skoch, A., Jirů, F., Hlustik, P., Martinkova, P., Zvara, K. and Rasova, K. (2011) Fractional anisotropy and mean diffusivity in the corpus callosum of patients with multiple sclerosis: The effect of physiotherapy. Neuroradiology 53, 917-926.

Jiang, G., Yin, X., Li, C., Li, L., Zhao, L., Evans, A. C., Jiang, T., Wu, J. and Wang, J. (2015) The plasticity of brain gray matter and white matter following lower limb amputation. Neural Plasticity 2015, 1-10. Jo, S., Yarishkin, O., Hwang, Y. J., Chun, Y. E., Park, M., Woo, D. H., Bae, J. Y., Kim, T., Lee, J., Chun, H., Park, H. J., Lee, D. Y., Hong, J., Kim, H. Y., Oh, S., Park, S. J., Lee, H., Yoon, B., Kim, Y., Jeong, Y., Shim, I., Bae, Y. C., Cho, J., Kowall, N. W., Ryu, H., Hwang, E., Kim, D. and Lee, C. J. (2014) GABA from reactive astrocytes impairs memory in mouse models of Alzheimer's disease. Nature Medicine 20, 886-896.

Jones, D. K., Knösche, T. R. and Turner, R. (2013) White matter integrity, fiber count, and other fallacies: The do's and don'ts of diffusion MRI. Neuroimage 73, 239-254.

Koerte, I. K. and Muehlmann, M. (2014) Diffusion tensor imaging. MRI in Psychiatry 50, 77-86.

Kee, N., Teixeira, C. M., Wang, A. H. and Frankland, P. W. (2007) Preferential incorporation of adult-generated granule cells into spatial memory networks in the dentate gyrus. Nature Neuroscience 10, 355-362.

Kilgard, M. P. (1998) Cortical map reorganization enabled by nucleus basalis activity. Science 279, 1714-1718.

Kingsley, P. B. (2006) Introduction to diffusion tensor imaging mathematics: Part I. Tensors, rotations, and eigenvectors. Concepts in Magnetic Resonance Part $A$ 28A, 101-122.

Kizil, C., Kaslin, J., Kroehne, V. and Brand, M. (2012) Adult neurogenesis and brain regeneration in zebrafish. Developmental Neurobiology 72, 429-461.

Kornack, D. R. and Rakic, P. (1999) Continuation of neurogenesis in the hippocampus of the adult macaque monkey. Proceedings of the National Academy of Sciences 96, 5768-5773.

Krubitzer, L. and Dooley, J. C. (2013) Cortical plasticity within and across lifetimes: How can development inform us about phenotypic transformations? Frontiers in Human Neuroscience 7, 620.

La Rosa, C. and Bonfanti, L. (2018) Brain plasticity in mammals: An example for the role of comparative medicine in the neurosciences. Frontiers in Veterinary Science 5, 274.

LaDage, L. D., Roth, T. C., Downs, C. J., Sinervo, B. and Pravosudov, V. V. (2017) Increased testosterone decreases medial cortical volume and neurogenesis in territorial side-blotched lizards (Uta stansburiana). Frontiers in Neuroscience 11, 97.

Laitinen, T., Sierra, A., Pitkänen, A. and Gröhn, O. (2010) Diffusion tensor MRI of axonal plasticity in the rat hippocampus. Neuroimage 51, 521530.

Lazarov, O. and Hollands, C. (2016) Hippocampal neurogenesis: Learning to remember. Progress in Neurobiology 138-140, 1-18.

Lazarov, O. and Marr, R. A. (2010) Neurogenesis and Alzheimer's disease: At the crossroads. Experimental Neurology 223, 267-281.

Lazic, S. E., Grote, H. E., Blakemore, C., Hannan, A. J., van Dellen, A., Phillips, W. and Barker, R. A. (2006) Neurogenesis in the R6/1 transgenic mouse model of Huntington's disease: Effects of environmental enrichment. European Journal of Neuroscience 23, 1829-1838.

Leuner, B. and Gould, E. (2010) Structural plasticity and hippocampal function. Annual Review of Psychology 61, 111-140.

Lévy, F., Batailler, M., Meurisse, M. and Migaud, M. (2017) Adult neurogenesis in sheep: Characterization and contribution to reproduction and behavior. Frontiers in Neuroscience 11, 570.

Li, W., Yu, J., Liu, Y., Huang, X., Abumaria, N., Zhu, Y., Huang, X., Xiong, W., Ren, C., Liu, X., Chui, D. and Liu, G. (2014) Elevation of brain magnesium prevents synaptic loss and reverses cognitive deficits in Alzheimer's disease mouse model. Molecular Brain 7, 65.

Liang, Y. Y., Allen Q., Chen, W., Gatto, R. G., Colon-Perez, L., Mareci, T. H. and Magin, R. L. (2016) A fractal derivative model for the characterization of anomalous diffusion in magnetic resonance imaging. Communications in Nonlinear Science and Numerical Simulation 39, 529-537.

Little, D. M. and Holloway, R. G. (2007) Diffusion tensor imaging: Scientific advance, clinical tool, or just a pretty picture? Neurology $\mathbf{6 8}$, 9-10.

Liu, J., Dietz, K., DeLoyht, J. M., Pedre, X., Kelkar, D., Kaur, J., Vialou, V., Lobo, M. K., Dietz, D. M., Nestler, E. J., Dupree, J. and Casaccia, P. (2012) Impaired adult myelination in the prefrontal cortex of socially isolated mice. Nature Neuroscience 15, 1621-1623. 
Liu, S. and Friel, D. D. (2008) Impact of the leaner/Q-type $\mathrm{Ca}^{2+}$ channel mutation on excitatory synaptic transmission in cerebellar Purkinje cells. The Journal of Physiology 586, 4501-4515.

Lobato, R. D. (2008) Historical vignette of Cajal's work "Degeneration and regeneration of the nervous system" with a reflection of the author. NeurocirugÍa 19, 456-468.

Low, L. K. and Cheng, H. (2006) Axon pruning: An essential step underlying the developmental plasticity of neuronal connections. Philosophical Transactions of the Royal Society B: Biological Sciences 361, 1531-1544.

Luzzati, F. (2006) Neurogenesis in the caudate nucleus of the adult rabbit. Journal of Neuroscience 26, 609-621.

Magin, R. L., Abdullah, O., Baleanu, D. and Zhou, X. J. (2008) Anomalous diffusion expressed through fractional order differential operators in the Bloch-Torrey equation. Journal of Magnetic Resonance 190, 255270.

Magistretti, P. J., Geisler, F. H., Schneider, J. S., Li, P. A., Fiumelli, H. and Sipione, S. (2019) Gangliosides: Treatment avenues in neurodegenerative disease. Frontiers in Neurology 10, 859.

Maine, A. R., Powers, S. D. and Lutterschmidt, D. I. (2014) Seasonal variation in cell proliferation and cell migration in the brain of adult redsided garter snakes (Thamnophis sirtalis parietalis). Brain, Behavior and Evolution 84, 181-196.

Mandolesi, L., Gelfo, F., Serra, L., Montuori, S., Polverino, A. and Curcio, G. (2017) Environmental factors promoting neural plasticity: Insights from animal and human studies. Neural Plasticity 2017, 1-10.

Manto, M. and Marmolino, D. (2009) Animal models of human cerebellar ataxias: A cornerstone for the therapies of the twenty-first century. The Cerebellum 8, 137-154.

Margotta, V. (2014) Relationships between seasonal thermal variations and cell proliferation in heterothermic vertebrates, as revealed by PCNA expression in the brain of adult Podarcis sicula. Italian Journal of Anatomy and Embryology 119, 29-37.

Martin, A. R., De Leener, B., Cohen-Adad, J., Cadotte, D. W., Kalsi-Ryan, S., Lange, S. F., Tetreault, L., Nouri, A., Crawley, A., Mikulis, D. J., Ginsberg, H. and Fehlings, M. G. (2017) A novel MRI biomarker of spinal cord white matter injury: $\mathrm{T} 2 *$-weighted white matter to gray matter signal intensity ratio. American Journal of Neuroradiology 38, 1266-1273.

Martínez-Cerdeño, V., Cunningham, C. L., Camacho, J., Antczak, J. L., Prakash, A. N., Cziep, M. E., Walker, A. I. and Noctor, S. C. (2012) Comparative analysis of the subventricular zone in rat, ferret and macaque: Evidence for an outer subventricular zone in rodents. PLoS One 7, e30178.

Masliah, E. R., E. Veinbergs, I., Mallory, M., Hashimoto, M., Takeda, A., Sagara, Y., Sisk, A. and Mucke, L. (2000) Dopaminergic loss and inclusion body formation in alpha-synuclein mice: Implications for neurodegenerative disorders. Science 287, 1265-1269.

Mattson, M. P. (2008) Glutamate and neurotrophic factors in neuronal plasticity and disease. Annals of the New York Academy of Sciences 1144, 97-112.

McColgan, P., Joubert, J., Tabrizi, S. J. and Rees, G. (2020) The human motor cortex microcircuit: Insights for neurodegenerative disease. $\mathrm{Na}$ ture Reviews Neuroscience 21, 401-415.

McGoldrick, P., Joyce, P. I., Fisher, E. M. C. and Greensmith, L. (2013) Rodent models of amyotrophic lateral sclerosis. Biochimica Et Biophysica Acta (BBA) - Molecular Basis of Disease 1832, 1421-1436.

Menke, R. A. L., Proudfoot, M., Wuu, J., Andersen, P. M., Talbot, K., Benatar, M. and Turner, M. R. (2016) Increased functional connectivity common to symptomatic amyotrophic lateral sclerosis and those at genetic risk. Journal of Neurology, Neurosurgery and Psychiatry 87, 580-588.

Mensch, S., Baraban, M., Almeida, R., Czopka, T., Ausborn, J., El Manira, A. and Lyons, D. A. (2015) Synaptic vesicle release regulates myelin sheath number of individual oligodendrocytes in vivo. Nature Neuroscience 18, 628-630.

Michaelis, E. K. (1998) Molecular biology of glutamate receptors in the central nervous system and their role in excitotoxicity, oxidative stress and aging. Progress in Neurobiology 54, 369-415.

Michou, E., Williams, S., Vidyasagar, R., Downey, D., Mistry, S., Edden,
R. A. E. and Hamdy, S. (2015) fMRI and MRS measures of neuroplasticity in the pharyngeal motor cortex. Neuroimage 117, 1-10.

Mimura, K., Oga, T., Sasaki, T., Nakagaki, K., Sato, C., Sumida, K., Hoshino, K., Saito, K., Miyawaki, I., Suhara, T., Aoki, I., Minamimoto, T. and Ichinohe, N. (2019) Abnormal axon guidance signals and reduced interhemispheric connection via anterior commissure in neonates of marmoset ASD model. Neuroimage 195, 243-251.

Mole, J. P., Subramanian, L., Bracht, T., Morris, H., Metzler-Baddeley, C. and Linden, D. E. J. (2016) Increased fractional anisotropy in the motor tracts of Parkinson's disease suggests compensatory neuroplasticity or selective neurodegeneration. European Radiology 26, 3327-3335.

Monje, M. (2018) Myelin plasticity and nervous system function. Annual Review of Neuroscience 41, 61-76.

Morfini, G. A., Bosco, D. A., Brown, H., Gatto, R., Kaminska, A., Song, Y., Molla, L., Baker, L., Marangoni, M. N., Berth, S., Tavassoli, E., Bagnato, C., Tiwari, A., Hayward, L. J., Pigino, G. F., Watterson, D. M., Huang, C., Banker, G., Brown, R. H. and Brady, S. T. (2013) Inhibition of fast axonal transport by pathogenic SOD1 involves activation of p38 MAP kinase. PLoS One 8, e65235.

Mori, S., Crain, B. J., Chacko, V. P. and Van Zijl, P. C. M. (1999) Threedimensional tracking of axonal projections in the brain by magnetic resonance imaging. Annals of Neurology 45, 265-269.

Mori, S. and Zhang, J. (2006) Principles of diffusion tensor imaging and its applications to basic neuroscience research. Neuron 51, 527-539.

Murphy, T., Dias, G. P. and Thuret, S. (2014) Effects of diet on brain plasticity in animal and human studies: Mind the gap. Neural Plasticity 2014, 1-32.

Näslund, J., Larsen, M. H., Thomassen, S. T., Aarestrup, K. and Johnsson, J. I. (2017) Environment-dependent plasticity and ontogenetic changes in the brain of hatchery-reared Atlantic salmon. Journal of Zoology 301, 75-82.

Noppeney, U., Friston, K. J., Ashburner, J., Frackowiak, R. and Price, C. J. (2005) Early visual deprivation induces structural plasticity in gray and white matter. Current Biology 15, R488-R490.

Nuber, S., Petrasch-Parwez, E., Winner, B., Winkler, J., von Horsten, S., Schmidt, T., Boy, J., Kuhn, M., Nguyen, H. P., Teismann, P., Schulz, J. B., Neumann, M., Pichler, B. J., Reischl, G., Holzmann, C., Schmitt, I., Bornemann, A., Kuhn, W., Zimmermann, F., Servadio, A. and Riess, O. (2008) Neurodegeneration and motor dysfunction in a conditional model of Parkinson's disease. Journal of Neuroscience 28, 2471-2484.

Olsen, D. P. and Keshishian, H. (2012) Experimental methods for examining synaptic plasticity in drosophila. Cold Spring Harbor Protocols 2012, pdb.top067785-pdb.top067785.

Ota, Y., Zanetti, A. T. and Hallock, R. M. (2013) The role of astrocytes in the regulation of synaptic plasticity and memory formation. Neural Plasticity 2013, 1-11.

Pang, T. Y. C., Stam, N. C., Nithianantharajah, J., Howard, M. L. and Hannan, A. J. (2006) Differential effects of voluntary physical exercise on behavioral and brain-derived neurotrophic factor expression deficits in Huntington's disease transgenic mice. Neuroscience 141, 569-584.

Parolisi, R., Cozzi, B. and Bonfanti, L. (2018) Humans and dolphins: Decline and fall of adult neurogenesis. Frontiers in Neuroscience 12, 497.

Payne, B. R. (2004) Neuroplasticity in the cat's visual system: Test of the role of the expanded retino-geniculo-parietal pathway in behavioral sparing following early lesions of visual cortex. Experimental Brain Research 155, 69-80.

Péran, P., Nemmi, F., Dutilleul, C., Finamore, L., Falletta Caravasso, C., Troisi, E., Iosa, M., Sabatini, U. and Grazia Grasso, M. (2020) Neuroplasticity and brain reorganization associated with positive outcomes of multidisciplinary rehabilitation in progressive multiple sclerosis: A fMRI study. Multiple Sclerosis and Related Disorders 42, 102127.

Phelps, E. A. and LeDoux, J. E. (2005) Contributions of the amygdala to emotion processing: From animal models to human behavior. Neuron 48, 175-187.

Phillips, W. (2005) Abnormalities of neurogenesis in the R6/2 mouse model of Huntington's disease are attributable to the in vivo microenvironment. Journal of Neuroscience 25, 11564-11576.

Piumatti, M., Palazzo, O., La Rosa, C., Crociara, P., Parolisi, R., Luzzati, F., Lévy, F. and Bonfanti, L. (2018) Non-newly generated, "immature" 
neurons in the sheep brain are not restricted to cerebral cortex. The Journal of Neuroscience 38, 826-842.

Poldrack, R. A. (2000) Imaging brain plasticity: Conceptual and methodological issues- a theoretical review. Neuroimage 12, 1-13.

Ponti, G., Crociara, P., Armentano, M. and Bonfanti, L. (2010) Adult neurogenesis without germinal layers: The "atypical" cerebellum of rabbits. Archives Italiennes de Biologie 148, 147-158.

Ponti, G., Peretto, P. and Bonfanti, L. (2008) Genesis of neuronal and glial progenitors in the cerebellar cortex of peripuberal and adult rabbits. PLoS One 3, e2366.

Popov, A., Denisov, P., Bychkov, M., Brazhe, A., Lyukmanova, E., Shenkarev, Z., Lazareva, N., Verkhratsky, A. and Semyanov, A. (2020) Caloric restriction triggers morphofunctional remodeling of astrocytes and enhances synaptic plasticity in the mouse hippocampus. Cell Death and Disease 11, 208.

Porto, F. H. D. G., Fox, A. M., Tusch, E. S., Sorond, F., Mohammed, A. H. and Daffner, K. R. (2015) In vivo evidence for neuroplasticity in older adults. Brain Research Bulletin 114, 56-61.

Powell, F., Tosun, D., Sadeghi, R., Weiner, M., Raj, A. and the Alzheimer's Disease Neuroimaging Initiative (2018) Preserved structural network organization mediates pathology spread in Alzheimer's disease spectrum despite loss of white matter tract integrity. Journal of Alzheimer's Disease 65, 747-764.

Powers, A. S. (2016) Plasticity and adult neurogenesis in amphibians and reptiles: More questions than answers. Brain, Behavior and Evolution 87, 175-183.

Purger, D., Gibson, E. M. and Monje, M. (2016) Myelin plasticity in the central nervous system. Neuropharmacology 110, 563-573.

Quallo, M. M., Price, C. J., Ueno, K., Asamizuya, T., Cheng, K., Lemon, R. N. and Iriki, A. (2009) Gray and white matter changes associated with tool-use learning in macaque monkeys. Proceedings of the $\mathrm{Na}$ tional Academy of Sciences of the United States of America 106, 18379. 18384

Rattray, I., Smith, E. J., Crum, W. R., Walker, T. A., Gale, R., Bates, G. P. and Modo, M. (2013) Correlations of behavioral deficits with brain pathology assessed through longitudinal MRI and histopathology in the R6/1 mouse model of Huntington's disease. PLoS One 8, e84726.

Richardson, P. M. (1994) Ciliary neurotrophic factor: A review. Pharmacology and Therapeutics 63, 187-198.

Rinaldo, L. and Hansel, C. (2010) Ataxias and cerebellar dysfunction: Involvement of synaptic plasticity deficits? Functional Neurology $\mathbf{2 5}$, 135-139.

Rumpel, S., Hatt, H. and Gottmann, K. (1998) Silent synapses in the developing rat visual cortex: Evidence for postsynaptic expression of synaptic plasticity. The Journal of Neuroscience 18, 8863-8874.

Saarma, M. and Sariola, H. (1999) Other neurotrophic factors: Glial cell line-derived neurotrophic factor (GDNF). Microscopy Research and Technique 45, 292-302.

Sama, R. R. K., Fallini, C., Gatto, R., McKeon, J. E., Song, Y., Rotunno, M. S., Penaranda, S., Abdurakhmanov, I., Landers, J. E., Morfini, G., Brady, S. T. and Bosco, D. A. (2017) ALS-linked FUS exerts a gain of toxic function involving aberrant p38 MAPK activation. Scientific Reports 7, 115 .

Sampedro, C., Font, E. and Desfilis, E. (2008) Size variation and cell proliferation in chemosensory brain areas of a lizard (Podarcis hispanica): Effects of sex and season. European Journal of Neuroscience 28, 87 98

Schilling, K., Gao, Y., Janve, V., Stepniewska, I., Landman, B. A. and Anderson, A. W. (2017) Can increased spatial resolution solve the crossing fiber problem for diffusion MRI? NMR in Biomedicine 30, e3787.

Schlachetzki, J. C. M., Saliba, S. W. and Oliveira, A. C. P. D. (2013) Studying neurodegenerative diseases in culture models. Revista Brasileira De Psiquiatria 35, S92-S100.

Schnaar, R. L. (2019) The Biology of gangliosides. Advances in Carbohydrate Chemistry and Biochemistry 76, 113-148.

Scholz, J., Niibori, Y., W Frankland, P. and P Lerch, J. (2015) Rotarod training in mice is associated with changes in brain structure observable with multimodal MRI. Neuroimage 107, 182-189.

Serita, T., Miyahara, M., Tanimizu, T., Takahashi, S., Oishi, S., Na- gayoshi, T., Tsuji, R., Inoue, H., Uehara, M. and Kida, S. (2019) Dietary magnesium deficiency impairs hippocampus-dependent memories without changes in the spine density and morphology of hippocampal neurons in mice. Brain Research Bulletin 144, 149-157.

Shany-Ur, T. and Rankin, K. P. (2011) Personality and social cognition in neurodegenerative disease. Current Opinion in Neurology 24, 550-555.

Sheppard, P. A. S., Choleris, E. and Galea, L. A. M. (2019) Structural plasticity of the hippocampus in response to estrogens in female rodents. Molecular Brain 12, 22

Siwak-Tapp, C. T., Head, E., Muggenburg, B. A., Milgram, N. W. and Cotman, C. W. (2007) Neurogenesis decreases with age in the canine hippocampus and correlates with cognitive function. Neurobiology of Learning and Memory 88, 249-259.

Smart, I. H. M. (2002) Unique morphological features of the proliferative zones and postmitotic compartments of the neural epithelium giving rise to striate and extrastriate cortex in the monkey. Cerebral Cortex 12, 37-53.

Smith, C. C., Gibbs, T. T. and Farb, D. H. (2014) Pregnenolone sulfate as a modulator of synaptic plasticity. Psychopharmacology 231, 35373556 .

Smith, J. C., Lancaster, M. A., Nielson, K. A., Woodard, J. L., Seidenberg, M., Durgerian, S., Sakaie, K. and Rao, S. M. (2016) Interactive effects of physical activity and APOE- $\varepsilon 4$ on white matter tract diffusivity in healthy elders. Neuroimage 131, 102-112.

Soares, J. M., Marques, P., Alves, V. and Sousa, N. (2013) A hitchhiker's guide to diffusion tensor imaging. Frontiers in Neuroscience 7, 31.

Soma, K. K., Tramontin, A. D., Featherstone, J. and Brenowitz, E. A. (2004) Estrogen contributes to seasonal plasticity of the adult avian song control system. Journal of Neurobiology 58, 413-422.

Song, S. K., Sun, S. W., Ju, W. K., Lin, S. J., Cross, A. H. and Neufeld A. H. (2003) Diffusion tensor imaging detects and differentiates axon and myelin degeneration in mouse optic nerve after retinal ischemia Neuroimage 20, 1714-1722.

Sousa, N., Cerqueira, J. J. and Almeida, O. F. X. (2008) Corticosteroid receptors and neuroplasticity. Brain Research Reviews 57, 561-570.

Stahnisch, F. W. and Nitsch, R. (2002) Santiago Ramón y Cajal's concept of neuronal plasticity: The ambiguity lives on. Trends in Neurosciences 25, 589-591.

Stassart, R. M., Möbius, W., Nave, K. and Edgar, J. M. (2018) The axonmyelin unit in development and degenerative disease. Frontiers in Neuroscience 12, 467.

Sumiyoshi, A., Taki, Y., Nonaka, H., Takeuchi, H. and Kawashima, R. (2014) Regional gray matter volume increases following 7days of voluntary wheel running exercise: A longitudinal VBM study in rats. Neuroimage $\mathbf{9 8}, 82-90$.

Sun, B., Halabisky, B., Zhou, Y., Palop, J. J., Yu, G., Mucke, L. and Gan, L. (2009) Imbalance between GABAergic and glutamatergic transmission impairs adult neurogenesis in an animal model of Alzheimer's disease. Cell Stem Cell 5, 624-633.

Takumi, Y., Ramírez-León, V., Laake, P., Rinvik, E. and Ottersen, O. P. (1999) Different modes of expression of AMPA and NMDA receptors in hippocampal synapses. Nature Neuroscience 2, 618-624.

Tardif, C. L., Gauthier, C. J., Steele, C. J., Bazin, P., Schäfer, A., Schaefer, A., Turner, R. and Villringer, A. (2016) Advanced MRI techniques to improve our understanding of experience-induced neuroplasticity. Neuroimage 131, 55-72.

Tattersfield, A. S., Croon, R. J., Liu, Y. W., Kells, A. P., Faull, R. L. M. and Connor, B. (2004) Neurogenesis in the striatum of the quinolinic acid lesion model of Huntington's disease. Neuroscience 127, 319-332.

Thomas, C., Ye, F. Q., Irfanoglu, M. O., Modi, P., Saleem, K. S., Leopold, D. A. and Pierpaoli, C. (2014) Anatomical accuracy of brain connections derived from diffusion MRI tractography is inherently limited. Proceedings of the National Academy of Sciences 111, 16574-16579. pathogenesis of Parkinson's disease animal models from a molecular signaling perspective. Neural Plasticity 2020, 1-10.

Udovin, L., Quarracino, C., Herrera, M. I., Capani, F., Otero-Losada, M. and Perez-Lloret, S. (2020) Role of astrocytic dysfunction in the pathogenesis of Parkinson's disease animal models from a molecular signaling perspective. Neural Plasticity 2020, 1-10. 
Van Den Bosch, L. and Robberecht, W. (2008) Crosstalk between astrocytes and motor neurons: What is the message? Experimental Neurology 211, 1-6.

Van Ombergen, A., Laureys, S., Sunaert, S., Tomilovskaya, E., Parizel, P. M. and Wuyts, F. L. (2017) Spaceflight-induced neuroplasticity in humans as measured by MRI: What do we know so far? NPJ Microgravity $3,2$.

van Praag, H. (2005) Exercise enhances learning and hippocampal neurogenesis in aged mice. Journal of Neuroscience 25, 8680-8685.

Verkhratsky, A., Rodríguez, J. J. and Parpura, V. (2012) Neurotransmitters and integration in neuronal-astroglial networks. Neurochemical Research 37, 2326-2338.

von Horsten, S., Schmitt, I., Nguyen, H. P., Holzmann, C., Schmidt, T., Walther, T., Bader, M., Pabst, R., Kobbe, P., Krotova, J., Stiller, D., Kask, A., Vaarmann, A., Rathke-Hartlieb, S., Schulz, J. B., Grasshoff, U., Bauer, I., Vieira-Saecker, A. M. M., Paul, M., Jones, L., Lindenberg, K. S., Landwehrmeyer, B., Bauer, A., Li, X. and Riess, O. (2003) Transgenic rat model of Huntington's disease. Human Molecular Genetics 12, 617-624.

Vonsattel, J. P. G. (2007) Huntington disease models and human neuropathology: Similarities and differences. Acta Neuropathologica 115, 55-69.

Voss, M. W., Vivar, C., Kramer, A. F. and van Praag, H. (2013) Bridging animal and human models of exercise-induced brain plasticity. Trends in Cognitive Sciences 17, 525-544.

Voss, P., Thomas, M. E., Cisneros-Franco, J. M. and de Villers-Sidani, É. (2017) Dynamic brains and the changing rules of neuroplasticity: Implications for learning and recovery. Frontiers in Psychology 8, 1657.

Wang, W., Yu, J., Liu, Y., Yin, R., Wang, H., Wang, J., Tan, L., Radua, J. and Tan, L. (2015) Voxel-based meta-analysis of grey matter changes in Alzheimer's disease. Translational Neurodegeneration 4, 6.

Winner, B., Geyer, M., Couillard-Despres, S., Aigner, R., Bogdahn, U., Aigner, L., Kuhn, G. and Winkler, J. (2006) Striatal deafferentation increases dopaminergic neurogenesis in the adult olfactory bulb. $E x$ - perimental Neurology 197, 113-121.

Winner, B., Kohl, Z. and Gage, F. H. (2011) Neurodegenerative disease and adult neurogenesis. European Journal of Neuroscience 33, 11391151.

Winner, B. L., D. C., Rockenstein, E., Aigner, R., Aigner, L., Masliah, E., Kuhn, H. G. and Winkler, J. (2004) Human wild-type alpha-synuclein impairs neurogenesis. Journal of Neuropathology and Experimental Neurology 63, 1155-1166.

Winner, B. W., J. (2015) Adult neurogenesis in neurodegenerative diseases. Cold Spring Harbor Perspectives in Biology 7, a021287.

Xu, Z., Li, L., Bao, J., Wang, Z., Zeng, J., Liu, E., Li, X., Huang, R., Gao, D., Li, M., Zhang, Y., Liu, G. and Wang, J. (2014) Magnesium protects cognitive functions and synaptic plasticity in streptozotocin-induced sporadic Alzheimer's model. PLoS One 9, e108645.

Yamada, M., Onodera, M., Mizuno, Y. and Mochizuki, H. (2004) Neurogenesis in olfactory bulb identified by retroviral labeling in normal and 1-methyl-4-phenyl-1,2,3,6-tetrahydropyridine-treated adult mice. Neuroscience 124, 173-181.

Yiu, G. and He, Z. (2006) Glial inhibition of CNS axon regeneration. Nature Reviews Neuroscience 7, 617-627.

Zagrebelsky, M., Gödecke, N., Remus, A. and Korte, M. (2018) Cell typespecific effects of BDNF in modulating dendritic architecture of hippocampal neurons. Brain Structure and Function 223, 3689-3709.

Zatorre, R. J., Fields, R. D. and Johansen-Berg, H. (2012) Plasticity in gray and white: Neuroimaging changes in brain structure during learning. Nature Neuroscience 15, 528-536.

Zhang, H., Schneider, T., Wheeler-Kingshott, C. A. and Alexander, D. C. (2012) NODDI: Practical in vivo neurite orientation dispersion and density imaging of the human brain. Neuroimage 61, 1000-1016.

Zhang, X. and Poo, M. (2010) Progress in neural plasticity. Science China Life Sciences 53, 322-329.

Zupanc, G. K. H. (2008) Adult neurogenesis and neuronal regeneration in the brain of teleost fish. Journal of Physiology-Paris 102, 357-373. 